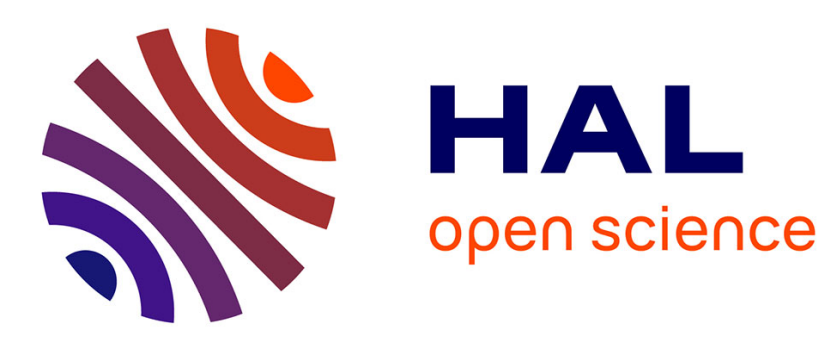

\title{
Nonparametric survival function estimation for data subject to interval censoring case 2
}

Olivier Bouaziz, Elodie Brunel, Fabienne Comte

\section{To cite this version:}

Olivier Bouaziz, Elodie Brunel, Fabienne Comte. Nonparametric survival function estimation for data subject to interval censoring case 2. Journal of Nonparametric Statistics, 2019, 31 (4), pp.952-987. 10.1080/10485252.2019.1669791. hal-01766456

\section{HAL Id: hal-01766456 https://hal.science/hal-01766456}

Submitted on 13 Apr 2018

HAL is a multi-disciplinary open access archive for the deposit and dissemination of scientific research documents, whether they are published or not. The documents may come from teaching and research institutions in France or abroad, or from public or private research centers.
L'archive ouverte pluridisciplinaire HAL, est destinée au dépôt et à la diffusion de documents scientifiques de niveau recherche, publiés ou non, émanant des établissements d'enseignement et de recherche français ou étrangers, des laboratoires publics ou privés. 


\title{
NONPARAMETRIC SURVIVAL FUNCTION ESTIMATION FOR DATA SUBJECT TO INTERVAL CENSORING CASE 2
}

\author{
OLIVIER BOUAZIZ ${ }^{(1)}$, ELODIE BRUNEL ${ }^{(2)}$, FABIENNE COMTE $^{(1)}$
}

\begin{abstract}
In this paper, we propose a new strategy of estimation for the survival function $S$, associated to a survival time subject to interval censoring case 2. Our method is based on a least squares contrast of regression type with parameters corresponding to the coefficients of the development of $S$ on an orthonormal basis. We obtain a collection of projection estimators where the dimension of the projection space has to be adequately chosen via a model selection procedure. For compactly supported bases, we obtain adaptive results leading to general nonparametric rates. However, our results can be used for non compactly supported bases, a true novelty in regression setting, and we use specifically the Laguerre basis which is $\mathbb{R}^{+}$-supported and thus well suited when nonnegative random variables are involved in the model. Simulation results comparing our proposal with previous strategies show that it works well in a very general context. A real data set is considered to illustrate the methodology.
\end{abstract}

MSC 2010 subject classification: $62 \mathrm{~N} 02-62 \mathrm{G} 05$

Keywords and phrases: Interval censoring, nonparametric estimation, regression contrast, survival function.

\section{INTRODUCTION}

Let $X_{1}$ be a survival time of interest (the time at which the event of interest occurs) with unknown survival function $S, S(x)=\mathbb{P}\left(X_{1}>x\right)$. Our aim is to propose a nonparametric estimator of $S$ in a setting where $X_{1}$ is not observed, but subject to interval censoring case 2 . To be more precise, the observations are $\left(L_{i}, U_{i}, \delta_{i}\right)_{1 \leq i \leq n}$ with

$$
\delta_{i}=\left\{\begin{array}{rll}
-1 & \text { if } & X_{i} \leq L_{i} \\
0 & \text { if } & L_{i}<X_{i} \leq U_{i} \\
1 & \text { if } & X_{i}>U_{i}
\end{array}\right.
$$

We assume that the triples $\left(L_{i}, U_{i}, \delta_{i}\right)_{1 \leq i \leq n}$ are i.i.d. and that the $\left(L_{i}, U_{i}\right)$ are independent of the $X_{i}$. Note that interval censoring case 1 corresponds to $U_{i}=L_{i}\left(\right.$ or $\left.L_{i}=-\infty\right)$, so that the $\delta_{i}$ s have only two modalities.

There have been previous proposals on the topic. First, Turnbull (1976) introduced an iterative procedure in order to obtain a Non Parametric Maximum Likelihood Estimator (NPMLE) of the survival function under different censoring and truncation types. Then Groeneboom and Wellner (1992) introduced the iterative convex minorant algorithm based on isotonic regression theory. Asymptotics of these estimators were also studied in Groeneboom and Wellner (1992) and asymptotics of functional estimators of the survival function were investigated in Geskus and Groeneboom $(1996,1997,1999)$. Among the problems, there was the question of building an explicit estimator reaching their rates, and Birgé (1999) solved it with an explicit histogram proposal for which he proved a $\mathbb{L}^{1}$-risk bound with adequate rate of order $(n \log (n))^{-1 / 3}$.

(1): MAP5, UMR 8145 CNRS, Université Paris Descartes, FRANCE, email: olivier.bouaziz@parisdescartes.fr, email: fabienne.comte@parisdescartes.fr

${ }^{(2)}$ : IMAG, Univ Montpellier, CNRS, Montpellier, France, email: ebrunel@math.univ-montp2.fr . 
Smooth estimators have also been proposed for interval censored data. In case 1, Yang (2000) studied the estimate of functionals of the survival function using locally linear smoothers and Brunel and Comte (2009) proposed two adaptive estimators, one of quotient type and another one of regression type, using projection methods. For interval censored data with case 2, spline methods were introduced in Kooperberg and Stone (1992) and a kernel method was studied in Braun et al. (2005) for the estimation of the density function. More recently, smooth alternatives to the NPMLE were proposed by using a kernel method in Groeneboom and Ketelaars (2011) and by introducing a log-concave constraint in the estimation procedure in Anderson-Bergman and $\mathrm{Yu}(2016)$.

Here, we propose a least squares contrast minimization method, in the spirit of Brunel and Comte (2009). First, we propose a procedure which relies on the regression equation $\mathbb{E}[1-$ $\left.\mathbb{1}_{\delta_{i}=-1} \mid L_{i}\right]=S\left(L_{i}\right)$ and another one based on its counterpart $\mathbb{E}\left[\mathbb{1}_{\delta_{i}=1} \mid U_{i}\right]=S\left(U_{i}\right)$. However, we want to elaborate a method taking both relations into account, and we finally propose a mixed contrast. We explain how it is built, and in what sense it improves the estimation.

The bases used in Brunel and Comte (2009) are compactly supported. This requires to define the domain of estimation at the very beginning of the procedure. This step is avoided by using the Laguerre basis, which is $\mathbb{R}^{+}$-supported. However, this non-compact feature is excluded from the theoretical framework of Brunel and Comte (2009), as well as from most other papers on nonparametric least squares regression (see e.g. Baraud, 2002). Therefore, we borrow elements from a recent work by Comte and Genon-Catalot (2018), to include this possibility in our results. We are then able to provide mean-square risk bounds for the resulting estimators, to compute general rates of convergence in the compactly supported case, and to propose a model selection device leading to an automatic bias variance tradeoff. Note that, similarly to Brunel and Comte (2009), we obtain a final estimator taking values between 1 and 0 and decreasing thanks to the procedure described in Chernozhukov et al. (2009), which is conveniently associated with a R-package Rearrangement.

The plan of the paper is the following. Section 2 describes the bases and projection spaces and explains the way estimators are built. Non asymptotic risk bounds are then proved, which allow to discuss asymptotic rates in a rather general setting. Section 3 develops the model selection strategy and the associated risk bounds. Then we show, in thorough simulation experiments presented in Section 4 that our estimator works well, especially when using the Laguerre basis, in comparison with the NPMLE implemented in the prodlim $\mathrm{R}$ package and the log-concave estimator proposed by Anderson-Bergman and $\mathrm{Yu}$ (2016) in the logconPH R package. Real interval censored data on HIV infections are analyzed in Section 5 using our estimator. Most proofs are gathered in Section 6.

\section{DEFINITION AND STUDY OF PROJECTION ESTIMATORS}

We first present the different bases associated with projection estimators defined in the sequel.

2.1. Projection spaces. Consider $\Sigma_{m}(I)=\operatorname{span}\left(\varphi_{0}, \ldots, \varphi_{m-1}\right)$ where $\left(\varphi_{j}\right)_{0 \leq j \leq m-1}$ constitutes an orthonormal basis $\left\langle\varphi_{j}, \varphi_{k}\right\rangle=\delta_{j, k}$ with respect to the scalar product $\langle u, v\rangle=\int_{I} u(x) v(x) d x$. The domain $I$ is the support of the basis and can be an interval $[a, b]$ which shall be taken equal to $[0,1]$ for simplicity in the examples below. We will also consider the case where $I=\mathbb{R}^{+}$which can be very convenient in this type of problems.

The examples of bases we have in mind are the following.

- Histogram basis with $I=[0,1]$, defined by $h_{j}(x)=\sqrt{m} \mathbb{1}_{[j / m,(j+1) / m[}$ for $j=0, \ldots, m-1$. They can be generalized to piecewise polynomials with given degree $r$, by rescaling $Q_{0}, \ldots, Q_{r}$ the Legendre basis on each sub-interval $[j / m,(j+1) / m[, j=0, \ldots, m-1$. 
- Trigonometric basis, $I=[0,1], t_{0}(x)=\mathbb{1}_{[0,1]}(x), t_{2 j-1}(x)=\sqrt{2} \cos (2 \pi j x) \mathbb{1}_{[0,1]}(x)$, $t_{2 j}(x)=\sqrt{2} \sin (2 \pi j x) \mathbb{1}_{[0,1]}(x)$, for $2 j \leq m-1$. Generally, $m$ is chosen odd and in this case $j=1, \cdots, \frac{m-1}{2}$.

- The Laguerre basis associated with $I=\mathbb{R}^{+}$is defined as follows. Consider the Laguerre polynomials $\left(P_{j}\right)$ and the Laguerre functions $\left(\ell_{j}\right)$ given by

$$
P_{j}(x)=\sum_{k=0}^{j}(-1)^{k}\left(\begin{array}{l}
j \\
k
\end{array}\right) \frac{x^{k}}{k !}, \quad \ell_{j}(x)=\sqrt{2} P_{j}(2 x) e^{-x} \mathbb{1}_{x \geq 0}, \quad j \geq 0 .
$$

The collection $\left(\ell_{j}\right)_{j \geq 0}$ constitutes a complete orthonormal system on $\mathbb{L}^{2}\left(\mathbb{R}^{+}\right)$, such that (see Abramowitz and Stegun, 1964) $\forall j \geq 0, \forall x \in \mathbb{R}^{+},\left|\ell_{j}(x)\right| \leq \sqrt{2}$. For any function $f \in$ $\mathbb{L}^{2}\left(\mathbb{R}^{+}\right)$, we can develop $f$ on the Laguerre basis with $f=\sum_{j \geq 0} a_{j}(f) \ell_{j}, a_{j}(f)=\left\langle f, \ell_{j}\right\rangle$.

The general notation for all these bases is $\left(\varphi_{j}\right)_{j}$. They all satisfy

$$
\forall m \in \mathbb{N} \backslash\{0\}, \quad \sup _{x \in I} \sum_{j=0}^{m-1} \varphi_{j}^{2}(x):=\left\|\sum_{j=0}^{m-1} \varphi_{j}^{2}\right\|_{\infty} \leq c_{\varphi}^{2} m,
$$

for some constant $c_{\varphi}>0$ depending on the basis only. For the histogram basis, and the trigonometric basis with odd $m$, we have $c_{\varphi}=1$ and for the Laguerre basis, $c_{\varphi}^{2}=2$.

The most important thing here is the following: the first two bases are compactly supported, and the last one is not. Most regression results hold with compactly supported bases, a case which is generally exclusively considered. In this work, we provide results in the setting of non compactly supported bases, and show empirically that the Laguerre basis is very relevant for survival function estimation. It has the advantage that we do not have to choose an estimation support for the basis and thus for the computation of the coefficients of the function in the basis.

Moreover, we mention that we estimate the survival function rather than the cumulative distribution function because we need the function under estimation to be possibly squareintegrable on $\mathbb{R}^{+}$, in order to use the Laguerre basis. Note that survival functions in all classical models are square-integrable on $\mathbb{R}^{+}$. For instance, $S(x)=P_{\lambda, k}(x) e^{-\lambda x} \mathbb{1}_{\mathbb{R}^{+}}(x)$ for a $\gamma(k, \lambda)$ density, $P_{\lambda, k}$ being a polynomial depending on $\lambda$ with degree $k-1, S(x)=e^{-(x / \lambda)^{k}} \mathbb{1}_{\mathbb{R}^{+}}(x)$ for a Weibull density with parameters $k, \lambda, S(x)=\left(x_{m} / x\right)^{k} \mathbb{1}_{\left[x_{m},+\infty[\right.}(x)$ for $x_{m}>0, k>1 / 2$ for a Pareto density, the Gompertz-Makeham density $S(x)=\exp \left\{-\lambda x-\frac{\alpha}{\beta}\left(e^{\beta x}-1\right)\right\} \mathbb{1}_{x \geq 0}$, for $\alpha, \beta, \lambda>0$, are square integrable.

2.2. Notation. Let $\left(L_{i}, U_{i}, \delta_{i}\right)_{1 \leq i \leq n}$ be a $n$-sample from model (1). We denote by $f_{U}$ and $f_{L}$ the densities of $U_{1}$ and $L_{1}$ and by $f_{(L, U)}$ the joint density of $\left(L_{1}, U_{1}\right)$. We denote by $\left(\varphi_{j}\right)_{0 \leq j \leq m-1}$ an orthonormal $\mathbb{L}^{2}(I, d x)$ basis as described in section 2.1 .

We also use all along the paper the following notation. For any measurable $I$-supported functions $\psi, \tilde{\psi}$, we define the weighted $\mathbb{L}^{2}\left(I, f_{Z}(x) d x\right)$-norms and scalar products, for $Z=L, U$,

$$
\|\psi\|_{Z}^{2}=\int \psi^{2}(x) f_{Z}(x) d x, \quad \text { and }\langle\psi, \tilde{\psi}\rangle_{Z}=\int \psi(x) \tilde{\psi}(x) f_{Z}(x) d x
$$

as soon as $\|\psi\|_{Z}^{2}<+\infty,\|\tilde{\psi}\|_{Z}^{2}<+\infty$, and their empirical counterparts:

$$
\|\psi\|_{n, Z}^{2}=\frac{1}{n} \sum_{i=1}^{n} \psi^{2}\left(Z_{i}\right), \quad\langle\psi, \tilde{\psi}\rangle_{n, Z}=\frac{1}{n} \sum_{i=1}^{n} \psi\left(Z_{i}\right) \tilde{\psi}\left(Z_{i}\right) .
$$

Clearly, $\mathbb{E}\left(\|\psi\|_{n, Z}^{2}\right)=\|\psi\|_{Z}^{2}$, and $\mathbb{E}\left(\langle\psi, \tilde{\psi}\rangle_{n, Z}\right)=\langle\psi, \tilde{\psi}\rangle_{Z}$ for $Z=L, U$. 
As classical in regression setting, the following matrices and vectors are useful:

$$
\begin{cases}\Phi_{m}^{(L)}=\left(\varphi_{j}\left(L_{i}\right)\right)_{1 \leq i \leq n, 1 \leq j \leq m}, & \vec{\delta}^{(L)}=\left(1-\mathbb{1}_{\delta_{i}=-1}\right)_{1 \leq i \leq n}=\left(1-\mathbb{1}_{X_{i} \leq L_{i}}\right)_{1 \leq i \leq n} \\ \Phi_{m}^{(U)}=\left(\varphi_{j}\left(U_{i}\right)\right)_{1 \leq i \leq n, 1 \leq j \leq m}, & \vec{\delta}^{(U)}=\left(\mathbb{1}_{\delta_{i}=1}\right)_{1 \leq i \leq n}=\left(1-\mathbb{1}_{X_{i} \leq U_{i}}\right)_{1 \leq i \leq n},\end{cases}
$$

and

$$
\Psi_{m, Z}=\left(\left\langle\varphi_{j}, \varphi_{k}\right\rangle_{Z}\right)_{1 \leq j, k \leq m}, \quad \widehat{\Psi}_{m, Z}=\left(\left\langle\varphi_{j}, \varphi_{k}\right\rangle_{n, Z}\right) \text { for } \quad Z=U, L .
$$

We have $\Psi_{m, Z}=\mathbb{E}\left(\widehat{\Psi}_{m, Z}\right)$ for $Z=L, U$ and

$$
\widehat{\Psi}_{m, L}=\frac{1}{n}{ }^{t} \Phi_{m}^{(L)} \Phi_{m}^{(L)}, \quad \widehat{\Psi}_{m, U}=\frac{1}{n}{ }^{t} \Phi_{m}^{(U)} \Phi_{m}^{(U)} .
$$

In the sequel, the norm associated to matrices is the operator norm $\|A\|_{\text {op }}$ defined as the square-root of the largest eigenvalue of the matrix ${ }^{t} A A$ (or $A^{t} A$ ). If $A$ is a square symmetric and nonnegative matrix (i.e. for all vector $\vec{x},{ }^{t} \vec{x} A x \geq 0$ ), then $\|A\|_{\text {op }}$ is simply the largest of the eigenvalues of $A$, which are all nonnegative.

In particular, $\Psi_{m, Z}$ and $\widehat{\Psi}_{m, Z}$ are symmetric nonnegative matrices. Indeed, for $Z=L, U$, we have ${ }^{t} \vec{a} \Psi_{m, Z} \vec{a}=\|t\|_{Z}^{2} \geq 0$ where $t=\sum_{j=0}^{m-1} a_{j} \varphi_{j}$, and ${ }^{t} \vec{a}=\left(a_{0}, \ldots, a_{m-1}\right)$; analogously, ${ }^{t} \vec{a} \widehat{\Psi}_{m, Z} \vec{a}=\|t\|_{n, Z}^{2} \geq 0$.

2.3. Two naive regression estimators. The first idea is to extend the strategy developed in Brunel and Comte (2009) in presence of case 1 interval censoring. Noticing that

$$
\mathbb{E}\left(1-\mathbb{1}_{\delta_{i}=-1} \mid L_{i}\right)=S\left(L_{i}\right)
$$

we can define

$$
\widehat{S}_{m}^{(L)}=\arg \min _{t \in \Sigma_{m}} \gamma_{n}^{(L)}(t), \quad \gamma_{n}^{(L)}(t)=\frac{1}{n} \sum_{i=1}^{n} t^{2}\left(L_{i}\right)-\frac{2}{n} \sum_{i=1}^{n}\left(1-\mathbb{1}_{\delta_{i}=-1}\right) t\left(L_{i}\right) .
$$

This corresponds to the least squares estimator associated with the regression model (8), where $S$ would be replaced by $S_{m}$, the projection of $S$ on $\Sigma_{m}$ and the $m$ explanatory variables would be $\left(\varphi_{j}\left(L_{i}\right)\right)_{1 \leq i \leq n}$ for $j=0, \ldots, m-1$. To understand why the estimator may be suitable, just compute the expectation of the criterion (which is also its almost sure limit when $n$ tends to infinity). We have

$$
\begin{aligned}
\mathbb{E}\left(\gamma_{n}^{(L)}(t)\right) & =\mathbb{E}\left[t^{2}\left(L_{1}\right)\right]-2 \mathbb{E}\left[\mathbb{E}\left(\left(1-\mathbb{1}_{X_{1} \leq L_{1}}\right) \mid L_{1}\right) t\left(L_{1}\right)\right]=\int_{I} t^{2}(x) f_{L}(x) d x-2 \mathbb{E}\left[S\left(L_{1}\right) t\left(L_{1}\right)\right] \\
& =\int_{I}(t(x)-S(x))^{2} f_{L}(x) d x-\int_{I} S^{2}(x) f_{L}(x) d x .
\end{aligned}
$$

Clearly, the resulting term is minimal for $t=S$ and thus, the minimizer of $\gamma_{n}^{(L)}$ is likely to asymptotically minimize $\|t-S\|_{L}^{2}$ and to be near of $S$.

Similarly, relying on the equality $\mathbb{E}\left(\mathbb{1}_{\delta_{i}=1} \mid U_{i}\right)=S\left(U_{i}\right)$, we can set

$$
\widehat{S}_{m}^{(U)}=\arg \min _{t \in \Sigma_{m}} \gamma_{n}^{(U)}(t), \quad \gamma_{n}^{(U)}(t)=\frac{1}{n} \sum_{i=1}^{n} t^{2}\left(U_{i}\right)-\frac{2}{n} \sum_{i=1}^{n} \mathbb{1}_{\delta_{i}=1} t\left(U_{i}\right) .
$$

Standard computations analogous to those in linear regression models yield to the following formula, for $\overrightarrow{\hat{a}}_{m}^{(Z)}={ }^{t}\left(\hat{a}_{0}^{(Z)}, \ldots, \hat{a}_{m-1}^{(Z)}\right), Z=L, U$, the coordinates of $\widehat{S}_{m}^{(Z)}$ in the basis $\left(\varphi_{j}\right)_{0 \leq j \leq m-1}$,

$$
\widehat{S}_{m}^{(Z)}(x)=\sum_{j=0}^{m-1} \hat{a}_{j}^{(Z)} \varphi_{j}(x) \text { with } \overrightarrow{\hat{a}}_{m}^{(Z)}=\left({ }^{t} \Phi_{m}^{(Z)} \Phi_{m}^{(Z)}\right)^{-1}{ }^{t} \Phi_{m}^{(Z)} \vec{\delta}^{(Z)}
$$


where $\Phi_{m}^{(Z)}, \vec{\delta}^{(Z)}$ are defined in (6), provided that ${ }^{t} \Phi_{m}^{(Z)} \Phi_{m}^{(Z)}$ is invertible. Note that, if the bases are compactly supported, their supports $I_{Z}$ for $Z=L, U$ depend on the support of the $L_{i}$ 's denoted by $\operatorname{supp}(L)$ or the one of the $U_{i}$ 's denoted by $\operatorname{supp}(U)$ : they are chosen such that $I_{Z} \subset \operatorname{supp}(Z)$ for $Z=L, U$. The estimation spaces are thus $\Sigma_{m}\left(I_{Z}\right)$, and the basis should inherit from the same index, but it is omitted for the sake of readability. For the Laguerre basis, the support of the basis is fixed, $I_{Z}=\mathbb{R}^{+}$.

We can prove the following results, for the two estimators $\widehat{S}_{m}^{(L)}$ and $\widehat{S}_{m}^{(U)}$, relying on this formula:

Proposition 1. For $Z=L, U$, assume that ${ }^{t} \Phi_{m}^{(Z)} \Phi_{m}^{(Z)}$ is invertible almost surely. Let $\widehat{S}_{m}^{(Z)}$ be the estimator of $S$ on $I_{Z}$ defined by coefficients $\overrightarrow{\hat{a}}_{m}^{(Z)}$ in the basis $\varphi_{0}, \ldots, \varphi_{m-1}$ as given by (11). Then denoting by $S_{I}=S \mathbb{1}_{I}$, we have

$$
\mathbb{E}\left(\left\|\widehat{S}_{m}^{(Z)}-S_{I_{Z}}\right\|_{n, Z}^{2}\right) \leq \inf _{t \in \Sigma_{m}\left(I_{Z}\right)}\left\|t-S_{I_{Z}}\right\|_{Z}^{2}+\frac{1}{4} \frac{m}{n} .
$$

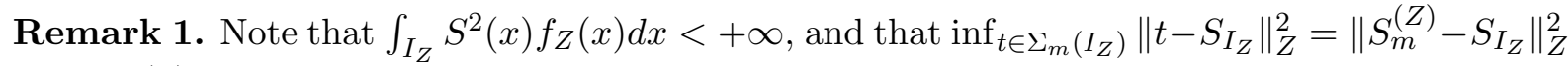
where $S_{m}^{(Z)}$ is the orthogonal projection of $S$ on $\Sigma_{m}\left(I_{Z}\right)$ with respect to the scalar product $\langle\cdot, \cdot\rangle_{Z}$ where $Z=L, U$. If moreover $S$ is square-integrable on $I_{Z} \cap \operatorname{supp}(Z)$ and $f_{L}$ and $f_{U}$ are upper bounded, by $f_{\max }^{(L)}$ and $f_{\max }^{(U)}$ respectively, we can recover a standard (non-weighted) $\mathbb{L}^{2}$-norm on $I_{Z} \cap \operatorname{supp}(Z)$ and get, for the bias term

$$
\inf _{t \in \Sigma_{m}}\left\|t-S_{I_{Z}}\right\|_{Z}^{2} \leq f_{\max }^{(Z)} \inf _{t \in \Sigma_{m}}\left\|\left(t-S_{I_{Z}}\right) \mathbf{1}_{\operatorname{supp}(Z)}\right\|^{2} \leq f_{\max }^{(Z)}\left\|S_{m}^{(Z)}-S_{I_{Z}}\right\|^{2}, \quad Z=L, U,
$$

where $S_{m}^{(Z)}$ is the standard orthogonal projection of $S_{I_{Z}}$ on $\Sigma_{m}\left(I_{Z}\right), S_{m}^{(Z)}=\sum_{j=0}^{m-1}\left\langle S, \varphi_{j}\right\rangle \varphi_{j}$.

Let us also briefly discuss about the invertibility assumption. First, in the case of the histogram basis, the matrix ${ }^{t} \Phi_{m}^{(Z)} \Phi_{m}^{(Z)}$ is diagonal (indeed in that case, $\varphi_{j} \varphi_{k} \equiv 0$ for $j \neq k$ ). It is thus invertible if no bin $[j / m,(j+1) / m$ [ is empty, and then explicit formula for the coefficients is available (see Section 2.6).

Moreover, asymptotically, for all bases, $(1 / n){ }^{t} \Phi_{m}^{(Z)} \Phi_{m}^{(Z)}$ tends to $\Psi_{m, Z}$ almost surely, for $Z=L, U$, when $n$ tends to infinity. We noticed that ${ }^{t} \vec{a} \Psi_{m, Z} \vec{a}=\|t\|_{Z}^{2}$ where $t=\sum_{j=0}^{m-1} a_{j} \varphi_{j}$, for $Z=L, U$. Assume that $I_{Z}$ is compact and $f_{Z}$ is lower bounded on $I_{Z}$ by $f_{0}^{(Z)}$. Then, for $t \neq 0,\|t\|_{Z}^{2} \geq f_{0}^{(Z)}\|t\|^{2}>0, Z=L, U$. Therefore, $\Psi_{m, Z}$ is invertible, which heuristically means that ${ }^{t} \Phi_{m}^{(Z)} \Phi_{m}^{(Z)}$ is "asymptotically" invertible.

Now, by using this strategy, we can see that we take separately two parts of the available information while we would like to take it completely. Moreover, the estimators will clearly perform well, but only either on the support of $L$ or on the one of $U$, and not on both. Their drawback can be easily illustrated, see Figure 1 for different choices of intervals for $\operatorname{supp}(L)$ and $\operatorname{supp}(U)$.

2.4. Improved estimator. Here, we explain our further investigations in order to obtain an estimator on a larger interval, in better accordance with all available date.

2.4.1. First step: estimator of differences. For $T(x, y)=\sum_{1 \leq j, k \leq m} a_{j, k} \varphi_{j}(x) \varphi_{k}(y)$ belonging to $\Sigma_{m} \otimes \Sigma_{m}$, we may also consider, as $\mathbb{E}\left(\mathbb{1}_{\delta_{i}=0} \mid U_{i}, L_{i}\right)=F\left(U_{i}\right)-\bar{F}\left(L_{i}\right)=S\left(L_{i}\right)-S\left(U_{i}\right)$, the contrast

$$
\frac{1}{n} \sum_{i=1}^{n} T^{2}\left(L_{i}, U_{i}\right)-\frac{2}{n} \sum_{i=1}^{n} \mathbb{1}_{\delta_{i}=0} T\left(L_{i}, U_{i}\right) .
$$


In that way, we would take all the observations into account. However, the resulting estimator would provide an estimator of the bi-variate function $G(x, y)=S(x)-S(y), x<y$, without taking its specific form into account: the underlying function is $S($.$) and it is univariate. However,$ due to the curse of dimensionality, the rate associated to the bidimensional problem would be bad, or at least worse than what we can expect for a univariate function. Now inserting in addition the specific form of $G$, we obtain

$$
\tilde{\gamma}_{n}(t)=\frac{1}{n} \sum_{i=1}^{n}\left[t\left(L_{i}\right)-t\left(U_{i}\right)\right]^{2}-\frac{2}{n} \sum_{i=1}^{n} \mathbb{1}_{\delta_{i}=0}\left[t\left(L_{i}\right)-t\left(U_{i}\right)\right]
$$

This contrast has expectation

$\mathbb{E}\left[\tilde{\gamma}_{n}(t)\right]=\iint[t(x)-t(y)-(S(x)-S(y))]^{2} f_{(L, U)}(x, y) d x d y-\iint(S(x)-S(y))^{2} f_{(L, U)}(x, y) d x d y$.

Here, we estimate $m$ coefficients, which may be relevant to recover $S$, except that the function is determined up to, at least, an additive constant. Now, the expectation can be re-written:

$$
\begin{aligned}
\mathbb{E}\left[\tilde{\gamma}_{n}(t)\right]= & \|t-S\|_{L}^{2}+\|t-S\|_{U}^{2}-2 \iint(t-S)(x)(t-S)(y) f_{(L, U)}(x, y) d x d y \\
& -\iint(S(x)-S(y))^{2} f_{(L, U)}(x, y) d x d y .
\end{aligned}
$$

The first two right-hand-side terms $\left(\left\|t-S_{I}\right\|_{L}^{2}+\left\|t-S_{I}\right\|_{U}^{2}\right)$ correspond to norms that we intend to simultaneously minimize, with $I \supseteq I_{L} \cup I_{U}$. The last term does not depend on the function $t$ and can be omitted. This is why we tried to kill the third term, of cross-product type. Noticing that

$$
\iint(t-S)(x)(t-S)(y) f_{(L, U)}(x, y) d x d y=\mathbb{E}\left[(t-S)\left(L_{1}\right)(t-S)\left(U_{1}\right)\right]
$$

and that, by conditioning by $\left(U_{i}, L_{i}\right)$, we have

$$
\mathbb{E}\left[\left(t\left(U_{i}\right)-\mathbb{1}_{\delta_{i}=1}\right)\left(t\left(L_{i}\right)-\mathbb{1}_{\delta_{i} \neq-1}\right)\right]=\mathbb{E}\left[\left(t\left(U_{i}\right)-S\left(U_{i}\right)\right)\left(t\left(L_{i}\right)-S\left(L_{i}\right)\right)\right]+\underbrace{\mathbb{E}\left[S\left(U_{i}\right)\left(1-S\left(L_{i}\right)\right)\right]}_{\text {independent of } t}
$$

we obtain an adequate term to add to the previous contrast.

2.4.2. New estimator. Thus, we corrected the contrast by replacing $\tilde{\gamma}_{n}(t)$ by

$$
\frac{1}{n} \sum_{i=1}^{n}\left[t\left(L_{i}\right)-t\left(U_{i}\right)\right]^{2}-\frac{2}{n} \sum_{i=1}^{n} \mathbb{1}_{\delta_{i}=0}\left[t\left(L_{i}\right)-t\left(U_{i}\right)\right]+\frac{2}{n} \sum_{i=1}^{n}\left(t\left(U_{i}\right)-\mathbb{1}_{\delta_{i}=1}\right)\left(t\left(L_{i}\right)-\mathbb{1}_{\delta_{i} \neq-1}\right) .
$$

This formula can be rewritten

$$
\|t\|_{n, U}^{2}+\|t\|_{n, L}^{2}-\frac{2}{n} \sum_{i=1}^{n} \mathbb{1}_{\delta_{i}=1} t\left(U_{i}\right)-\frac{2}{n} \sum_{i=1}^{n} \mathbb{1}_{\delta_{i} \neq-1} t\left(L_{i}\right)+\frac{2}{n} \sum_{i=1}^{n} \mathbb{1}_{\delta_{i}=1} .
$$

This is how we obtained our main contrast:

$$
\gamma_{n}(t)=\|t\|_{n, U}^{2}+\|t\|_{n, L}^{2}-\frac{2}{n} \sum_{i=1}^{n} \mathbb{1}_{\delta_{i}=1} t\left(U_{i}\right)-\frac{2}{n} \sum_{i=1}^{n} \mathbb{1}_{\delta_{i} \neq-1} t\left(L_{i}\right) .
$$

where $\|t\|_{n, U}^{2}$ and $\|t\|_{n, L}^{2}$ are defined by (5). We note that this contrast appears as the sum of the two previous ones and we straightforwardly obtain the following result.

Proposition 2. Using the norms defined in (4) and (5), we have

$$
\mathbb{E}\left(\gamma_{n}(t)\right)=\|t-S\|_{U}^{2}+\|t-S\|_{L}^{2}-\|S\|_{U}^{2}-\|S\|_{L}^{2} .
$$


Note that

$$
\|t-S\|_{U}^{2}+\|t-S\|_{L}^{2}=\int(t(x)-S(x))^{2}\left(f_{L}(x)+f_{U}(x)\right) d x:=\|t-S\|_{L+U}^{2} .
$$

Thus we obtain an estimator of $S$ on $I \supseteq I_{L} \cup I_{U}$. This means that if the estimation basis is compactly supported, the support must be chosen in accordance and is larger than for the two naive strategies, and for all the bases, the performance of the estimator may be good on this interval only.

Thus we define our final estimator by

$$
\widehat{S}_{m}=\arg \min _{t \in \Sigma_{m}(I)} \gamma_{n}(t)
$$

Assuming that ${ }^{t} \Phi_{m}^{(L)} \Phi_{m}^{(L)}+{ }^{t} \Phi_{m}^{(U)} \Phi_{m}^{(U)}$ is invertible, then the estimator can be computed as

$$
\widehat{S}_{m}=\sum_{j=1}^{m} \hat{a}_{j} \varphi_{j} \quad \overrightarrow{\hat{a}}_{m}=\left(\begin{array}{c}
\hat{a}_{0} \\
\vdots \\
\hat{a}_{m-1}
\end{array}\right)=\left[{ }^{t} \Phi_{m}^{(L)} \Phi_{m}^{(L)}+{ }^{t} \Phi_{m}^{(U)} \Phi_{m}^{(U)}\right]^{-1}\left({ }^{t} \Phi_{m}^{(L)} \vec{\delta}^{(L)}+{ }^{t} \Phi_{m}^{(U)} \vec{\delta}^{(U)}\right),
$$

where $\Phi_{m}^{(Z)}, \vec{\delta}^{(Z)}$ are defined in (6). This formula shows that the estimator uses all the data and is not the sum of the first two estimators.

The study of the estimator is more tedious, but it is interesting to see that we can prove the following result.

Proposition 3. Assume that ${ }^{t} \Phi_{m}^{(L)} \Phi_{m}^{(L)}+{ }^{t} \Phi_{m}^{(U)} \Phi_{m}^{(U)}$ is invertible almost surely. Then, for any $m \in\{1, \ldots, n\}$, we have

$$
\mathbb{E}\left(\left\|\widehat{S}_{m}-S_{I}\right\|_{n, U}^{2}+\left\|\widehat{S}_{m}-S_{I}\right\|_{n, L}^{2}\right) \leq \inf _{t \in \Sigma_{m}(I)}\left(\left\|t-S_{I}\right\|_{L+U}^{2}\right)+\frac{5}{2} \frac{m}{n} .
$$

We already noticed that $\int_{I} S^{2}(x)\left(f_{L}(x)+f_{U}(x)\right) d x<+\infty$. As announced, Proposition 3 shows that the estimator $\widehat{S}_{m}$ performs well on $I \cap(\operatorname{supp}(L) \cup \operatorname{supp}(U))$ due to the weight function $f_{L}+f_{U}$, $I \supseteq I_{L} \cup I_{U}$. In practice, for non localized bases such as Laguerre, the risk of the estimator is computed on $\left[\min \left(L_{i}\right), \max \left(U_{i}\right)\right]$. Note that the bias is now

$$
\inf _{t \in \Sigma_{m}(I)}\left(\left\|t-S_{I}\right\|_{U}^{2}+\left\|t-S_{I}\right\|_{L}^{2}\right)=\int\left(S_{m}(x)-S_{I}(x)\right)^{2}\left(f_{L}(x)+f_{U}(x)\right) d x
$$

where $S_{m}$ is the orthogonal projection of $S$ on $\Sigma_{m}(I)$ with respect to the scalar product $\langle., .\rangle_{L}+$ $\langle., .\rangle_{U}$. Following Remark 1, if $f_{L}$ and $f_{U}$ are bounded by $f_{\max }$, we get

$$
\inf _{t \in \Sigma_{m}(I)}\left(\left\|t-S_{I}\right\|_{U}^{2}+\left\|t-S_{I}\right\|_{L}^{2}\right) \leq 2 f_{\max }\left\|S_{m}-S_{I}\right\|^{2} .
$$

However, for compactly supported bases, the support $I$ is larger in the present setting than for the first two estimators, with the restriction that a hole between the supports of $L$ and $U$ may prevent the estimator to be computed. Indeed, think of the histogram case, empty bins imply that diagonal the matrix ${ }^{t} \Phi_{m}^{(L)} \Phi_{m}^{(L)}+{ }^{t} \Phi_{m}^{(U)} \Phi_{m}^{(U)}$ can not be inverted.

For Laguerre basis, $I=\mathbb{R}^{+}$, but the risk which is controlled corresponds to the risk on $\operatorname{supp}(L) \cup \operatorname{supp}(U)$. In practice, when considering this basis, a hole between the supports of $L$ and $U$ does not imply any practical problem in the procedure (see Figure 1). 
2.5. Discussion about rates. Inequalities provided in Proposition 1 and Proposition 3 can allow to compute convergence rates of the estimators.

- Consider the compactly supported bases described in Section 2.1 (such as histograms or piecewise polynomials). Assume that $f_{L}$ and $f_{U}$ are bounded on the support of the basis. The results stated in Brunel and Comte (2009), Corollary 3.1 p.8, apply here. They imply that the method provides convergent estimators $\widehat{S}_{m_{\mathrm{opt}}^{(Z)}}^{(Z)}$, with asymptotic rate $n^{-2 \alpha /(2 \alpha+1)}$ for $\alpha$ the Besov regularity of $S_{I_{Z}}$ when $S_{I_{Z}}$ belongs to a Besov ball, and $m_{\mathrm{opt}}^{(Z)}=O\left(n^{1 /(2 \alpha+1)}\right)$ for $Z=L, U$, and the same holds for $\widehat{S}_{m}$ on $I$. Those rates constitute a generalization of the rate $n^{-1 / 3}$ corresponding to $\alpha=1$ (rates obtained under Lipschitz type assumptions in Birgé (1999) to rates of order $n^{-\alpha /(2 \alpha+1)}$ for a general regularity $\alpha$ which can be larger than one for trigonometric bases or piecewise polynomials with degree $r \geq \alpha$. It is worth mentioning that Birgé (1999) presents an estimator reaching an improved rate (within a logarithmic factor) on a compact set and with an error measured in $\mathbb{L}^{1}$-distance, under a lower bound condition on the joint distribution $f_{(L, U)}$. It is known (see Comte and Genon-Catalot, 2018) that under a lower bound condition on $f_{U}$ and $f_{L}$ on $I$ (no hole case), we can extend our bounds to standard $\mathbb{L}^{2}$-risk on the support $I$.

- For $s \geq 0$, the Sobolev-Laguerre space with index $s$ (see Bongioanni and Torrea, 2009) is defined by:

$$
W^{s}=\left\{\theta: \mathbb{R}^{+} \rightarrow \mathbb{R}, \theta \in \mathbb{L}^{2}\left(\mathbb{R}^{+}\right),|\theta|_{s}^{2}:=\sum_{k \geq 0} k^{s} a_{k}^{2}(\theta)<+\infty\right\} .
$$

where $a_{k}(\theta)=\int_{\mathbb{R}^{+}} \theta(x) \ell_{k}(x) d x$. We define the ball $W^{s}(D)$ by

$$
W^{s}(D)=\left\{\theta \in W_{L}^{s},|\theta|_{s}^{2}=\sum_{k=0}^{\infty} k^{s} a_{k}^{2}(\theta) \leq D\right\} .
$$

For details on these spaces, and especially for regularity properties of functions in these spaces, we refer also the reader to Comte et al. (2015), Section 7.2. Now, if $f_{L}$ and $f_{U}$ are upper bounded on $I=\mathbb{R}^{+}$and $S_{I}$ belongs to $W^{s}(D)$, then the risks of $\widehat{S}_{m}^{(Z)}$ for $Z=L, U$ and $\widehat{S}_{m}$ can be bounded by $D m^{-s}+m /(2 n)$. Thus, choosing $m$ of order $n^{1 /(s+1)}$ yields a risk less than $n^{-s /(s+1)}$ and the estimators are therefore convergent. The interest of this basis is that the coefficient do not require any information on the estimation support; in censoring framework, this is an important advantage as the support is unknown.

2.6. Histogram case. In the specific case of histogram basis, the matrices $\Psi_{m, Z}, Z=L, U$, are diagonal and thus, invertibility conditions are easy to study and explicit formulas for the estimators can be given.

We take in this section $\varphi_{j}=h_{j}$ for $j=0, \ldots, m-1$, see Section 2.1. We define, the following cardinalities:

$$
N_{j}:=\operatorname{Card}\left\{i \in\{1, \ldots, n\}, L_{i} \in I_{j}\right\}, M_{j}:=\operatorname{Card}\left\{i \in\{1, \ldots, n\}, U_{i} \in I_{j}\right\},
$$

and

$N_{j}^{\prime}=\operatorname{Card}\left\{i \in\{1, \ldots, n\}, L_{i} \in I_{j}\right.$ and $\left.\delta_{i}=-1\right\}, M_{j}^{\prime}=\operatorname{Card}\left\{i \in\{1, \ldots, n\}, U_{i} \in I_{j}\right.$ and $\left.\delta_{i}=1\right\}$.

Then $\left\langle\varphi_{j}, \varphi_{k}\right\rangle_{n, U}=0$ if $j \neq k$ and $(m / n) M_{j}$ if $j=k$. So $\widehat{\Psi}_{m, U}=(m / n) \operatorname{diag}\left(M_{1}, \ldots, M_{m}\right)$. Analogously $\widehat{\Psi}_{m, L}=(m / n) \operatorname{diag}\left(N_{1}, \ldots, N_{m}\right)$. They are invertible if no $M_{j}$ is null for the first 
one, no $N_{j}$ is null for the second one. The estimator $\widehat{S}_{m}$ relies on the inversion of $\widehat{\Psi}_{m, L}+\widehat{\Psi}_{m, U}$ and is therefore possible if $M_{j}$ and $N_{j}$ are never simultaneously null. We obtain

$$
\hat{a}_{j}^{(L)}=\frac{1}{\sqrt{m}} \frac{N_{j}-N_{j}^{\prime}}{N_{j}}=\frac{1}{\sqrt{m}}\left(1-\frac{N_{j}^{\prime}}{N_{j}}\right), \quad \hat{a}_{j}^{(U)}=\frac{1}{\sqrt{m}} \frac{M_{j}^{\prime}}{M_{j}}, \quad \hat{a}_{j}=\frac{1}{\sqrt{m}} \frac{N_{j}-N_{j}^{\prime}+M_{j}^{\prime}}{M_{j}+N_{j}} .
$$

It is worth underlining that the last estimator in not the sum of the estimators $(\mathrm{L})$ and $(\mathrm{U})$, and it appears that all these estimators are different from Birgé's proposal. We explain in section 6.3 in what sense the estimator built from $\tilde{\gamma}_{n}$ may be more related to it.

\section{MOdEL SELECTION}

We propose here a model selection strategy for the estimator $\widehat{S}_{m}$, that is a data driven way of selecting $m$ from the data in a coherent way. Part of the tools we use here are inspired from the work on standard regression function estimation described developed in Comte and GenonCatalot (2018). They allow us to provide a generalization of the method presented in Brunel and Comte (2009) for interval censoring case I, and dedicated to the case of compactly supported bases. Note that a similar procedure would be possible for $\widehat{S}_{m}^{(Z)}, Z=L, U$, we experiment it numerically in section 4 , but do not give theoretical details.

To take into account both compactly and non compactly supported bases, we define the random collection of models as follows:

$$
\widehat{\mathcal{M}}_{n}=\left\{m \in \mathbb{N} \backslash\{0\}, m\left(\left\|\left(\widehat{\Psi}_{m, L}+\widehat{\Psi}_{m, U}\right)^{-1}\right\|_{\mathrm{op}}^{2} \vee 1\right) \leq 4 \mathfrak{c} \frac{n}{\log (n)}\right\},
$$

where

$$
\mathfrak{c}=\left(6 \wedge \frac{1}{\left\|f_{L}+f_{U}\right\|_{\infty}}\right) \frac{1}{48 c_{\varphi}^{2}},
$$

with $c_{\varphi}$ defined in (3). The theoretical (deterministic) counterpart is the set random sets $\mathcal{M}_{n}$ defined by

$$
\mathcal{M}_{n}=\left\{m \in \mathbb{N} \backslash\{0\}, m\left(\left\|\left(\Psi_{m, L}+\Psi_{m, U}\right)^{-1}\right\|_{\mathrm{op}}^{2} \vee 1\right) \leq \mathfrak{c} \frac{n}{\log (n)}\right\} .
$$

We propose to select the model following the rule:

$$
\hat{m}=\arg \min _{m \in \widehat{\mathcal{M}}_{n}}\left[\gamma_{n}\left(\widehat{S}_{m}\right)+\operatorname{pen}(m)\right]
$$

with pen $(m)=\kappa m / n$, and $\kappa$ a numerical constant. The constant $\kappa$ is calibrated on preliminary simulation experiments, and then fixed for the rest of the procedures. Relying on results stated in Comte and Genon-Catalot (2018), we can obtain the following result.

Theorem 1. Consider a nested collection of models $\left(\Sigma_{m}\right)_{m \in \mathcal{M}_{n}}$ with models satisfying (3) and $\mathcal{M}_{n}$ defined by (16), and the estimator defined by (12)-(13) and (17). Then there exists a value $\kappa_{0}>0$, such that $\forall \kappa \geq \kappa_{0}$,

$$
\mathbb{E}\left[\left\|\widehat{S}_{\hat{m}}-S_{I}\right\|_{L+U}^{2}\right] \leq C \inf _{m \in \mathcal{M}_{n}}\left(\inf _{t \in \Sigma_{m}}\left\|S_{I}-t\right\|_{L+U}^{2}+\frac{m}{n}\right)+\frac{C^{\prime}}{n}
$$

where $C$ is a numerical constant and $C^{\prime}$ is a constant depending on $f_{L}, f_{U}, \mathfrak{c}$.

The above inequalities show that the estimator makes an automatic bias-variance tradeoff, with a data driven selection criterion. The performance of $\widehat{S}_{\hat{m}}$ is valid on an interval which is larger than if $\widehat{S}_{m}^{(Z)}$ had been considered, for $Z=L$ or $U$. The loss of the procedure lies in the multiplicative constants $C$ (the nearer of 1 , the better), and in the restriction on the collection 

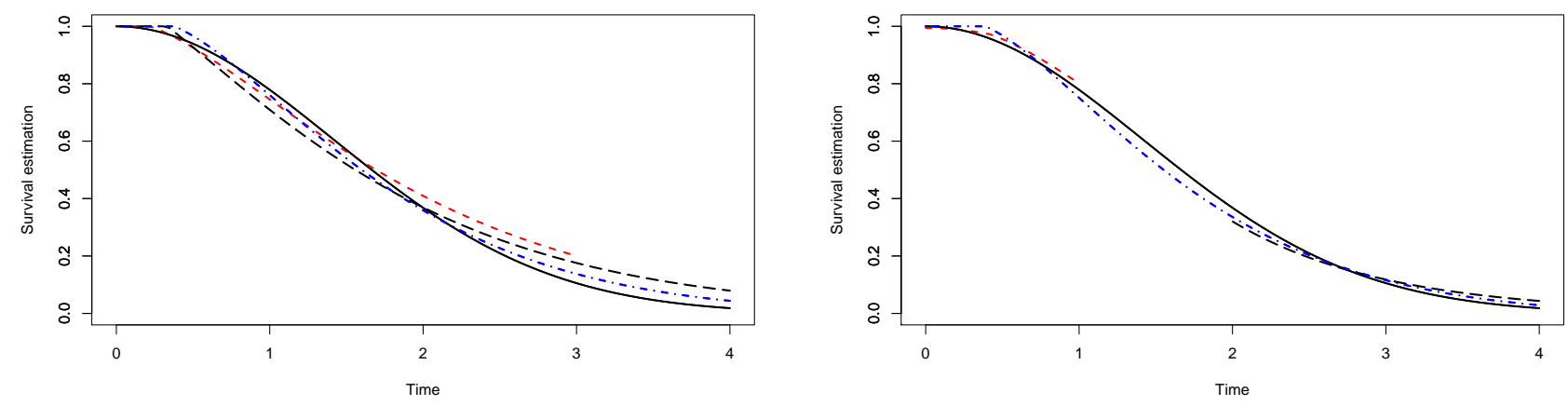

FiguRE 1. True survival curve (black solid line) $S_{I}$ for Model 1 and Laguerre basis estimators with sample size $n=1000: \widehat{S}_{\hat{m}_{L}}^{(L)}$ (red dashed line) built on $\operatorname{supp}(L), \widehat{S}_{\hat{m}_{U}}^{(U)}$ (black longdashed line) built on $\operatorname{supp}(U)$ and $\widehat{S}_{\hat{m}}$ (blue dotdashed line) built on $I=[0,4]$ on two different scenarios : on the left, scenario 1 with $\operatorname{supp}(L)=[0,3], \operatorname{supp}(U)=[0,4]$, on the right, scenario 4 with $\operatorname{supp}(L)=[0,1]$, $\operatorname{supp}(U)=[2,4]$.

given in (15) and (16), which must leave the optimal choice reachable. We discuss this in the next remark.

Remark 2. If the basis has compact support $I$ and $f_{U}$ and $f_{L}$ are lower bounded on $I$ by $f_{0}$, then we can prove

$$
\max \left(\left\|\Psi_{m, U}^{-1}\right\|_{\mathrm{op}}^{2},\left\|\Psi_{m, L}^{-1}\right\|_{\mathrm{op}}^{2}\right) \leq 1 / f_{0}^{2} \quad \text { and } \quad\left\|\left(\Psi_{m, L}+\Psi_{m, U}\right)^{-1}\right\|_{\mathrm{op}}^{2} \leq 4 / f_{0}^{2} .
$$

Thus, under such assumptions, it turns out that condition (16) reduces to the set of models $m$ such that $m \leq C n / \log (n)$, which is a very weak constraint. This implies that the adaptive estimator automatically reaches the best possible rate on Besov-type regularity spaces (see the end of Sections 2.3 and 2.4) on the domain determined by the support of the basis.

In the case of non compactly supported bases, such as the Laguerre basis which works very well for survival function estimation, condition (16) imposes a real restriction on the collection of models. For optimality issues, theoretical examples and illustrations, we refer to Comte and Genon-Catalot (2018).

\section{Simulation StUdy}

Our aim is to compare our new penalized estimator, built using the Laguerre basis, with other competitors. We consider the log-concave Nonparametric Maximum Likelihood Estimator (NPMLE) of Anderson-Bergman and Yu (2016) implemented using the logconPH R package and the unconstrained NPMLE implemented using the prodlim $\mathrm{R}$ package.

We have to choose the constant $\kappa$ in the penalty term and a preliminary rough calibration over some models shows that a range of values between 2 and 4 would suit. We take $\kappa=4$ which is the largest value of the range, possibly corresponding to over-penalization but also ensuring stability of the estimators.

We simulated $K=100$ samples of size $n=300$ and $n=1000$ from the following event time distributions : 
- Model 1:X $\sim$ Weibull $(a, b)$ the survival function is $S(x)=\exp \left(-(x / b)^{a}\right)$ with the shape parameter $a=2$ and scale parameter $b=2$ corresponding to a log-concave distribution.

- Model 2: $X \sim \mathcal{W}$ eibull $(a, b)$ with shape parameter $a=0.5$ and scale parameter $b=2$ corresponding to a non log-concave distribution.

- Model 3:X is distributed as a $\mathcal{B e t a}^{\prime}(\alpha, \beta)$ a beta prime distribution or a beta of type II with survival function $S(x)=\int_{x}^{+\infty} u^{\alpha-1}(1+u)^{-\alpha-\beta} /(B(\alpha, \beta)) d u$ for $x \geq 0$ where $B(\alpha, \beta)=\int_{0}^{1} t^{\alpha-1}(1-t)^{\beta-1} d t$ is the beta function with $\alpha=5$ and $\beta=2$ two shape parameters.

- Model 4: $X=6 Z$ with $Z \sim \mathcal{B}$ eta $(2,5)$ a standard beta distribution admitting the density function $f(x)=\Gamma(a+b) /(\Gamma(a) \Gamma(b)) x^{a-1}(1-x)^{b-1}$ for $0 \leq x \leq 1$ with shape parameters $a=2$ and $b=5$.

Note that Model 1 and 4 correspond to log-concave distributions while Model 2 and 3 do not. We also investigate different schemes for the distribution of the inspection times $L$ and $U$ :

- scenario $1: L \sim \mathcal{U}([0,3])$ and $U=L+\mathcal{U}([0,1])$.

- scenario $2: L \sim \mathcal{U}([0,1])$ and $U=L+\mathcal{U}([0,3])$.

- scenario $3: L, U \sim \mathcal{U}([0,4])$ with the constraint $0 \leq U-L \leq 0.1$ so that the times $L$ and $U$ can be very close to each other.

- scenario $4 L \sim \mathcal{U}([0,1])$ and $U \sim \mathcal{U}([2,4])$. In this case, there is a hole between 1 and 2 . This scenario makes sense in the context of diseases with a long-distance follow-up care.

We illustrate how model selection performs for histogram and Laguerre bases on Figure 2 . But we choose to compare only our Laguerre estimator with competitors because histogram estimators need additional conventions in scenario 4 . Nevertheless, it behaves well on an estimation interval without empty bins as shown in Figure 4 (left).

To assess the numerical performance of our penalized Least Squares estimator and its competitors, we compute the Average Mean Squared Error over a grid. We define a grid $t_{1}, \cdots, t_{100}$ of 100 equispaced points on $I=\left[\min \left(L_{i}\right), \max \left(U_{i}\right)\right]$. It is not always possible to evaluate the value of the NPMLE on the right of the interval, as for any product-limit estimator, it is biased and does not go to zero if the greatest observed value of $U_{i}$ corresponds to $\delta_{i}=1$. So we made the choice to shorten the grid at the upper bound of the last step of the NPMLE. Roughly speaking, the grid is shrunken at $\max \left(U_{i}, \delta_{i}=1\right)$ instead of $\max \left(U_{i}\right)$. This choice is rather in favour of the NPMLE but does not degrade significantly the results for the other estimators as far as we see in preliminary trials. For each generated sample, we compute the Mean Squared Error for each estimator, on the truncated grid described above. Then, we average over the 100 replicated samples of size $n=300$ or $n=1000$. The values of the Average Mean Squared Error AMSE $\times 10^{-3}$ are presented in Table 1 in Appendix A. We also report the median error in parenthesis. Model 1 and 4 match with log-concave distributions and as expected the logconcave estimator of Anderson-Bergman and Yu gives the best results whatever the scenario for the inspection times is. However, our Least Squares estimator challenges the NPMLE especially for scenario 3 and 4. When the distribution is not log-concave with Model 2 and 3, the AndersonBergman and $\mathrm{Yu}$ estimator has always the worst error. Our Least Squares estimator seems to be less performant than the NPMLE for Model 2 and $n=300$ but performs definitely better in scenario 4. Even if it performs a little worse in mean for some models, the Least Squares estimator built with Laguerre basis has no important failure, contrary to both NPMLE estimators. In fact, on Figure 4, we illustrate typical bad behaviours of both constrained/unconstrained NPMLEs: the log-concave estimator is very bad for non log-concave distribution (Figure 4, left) while the unconstrained NPMLE performs badly for scenario 4 (Figure 4, right) when there is a hole between the supports of $L$ and $U$. So the Least Squares estimator seems to be overall the 

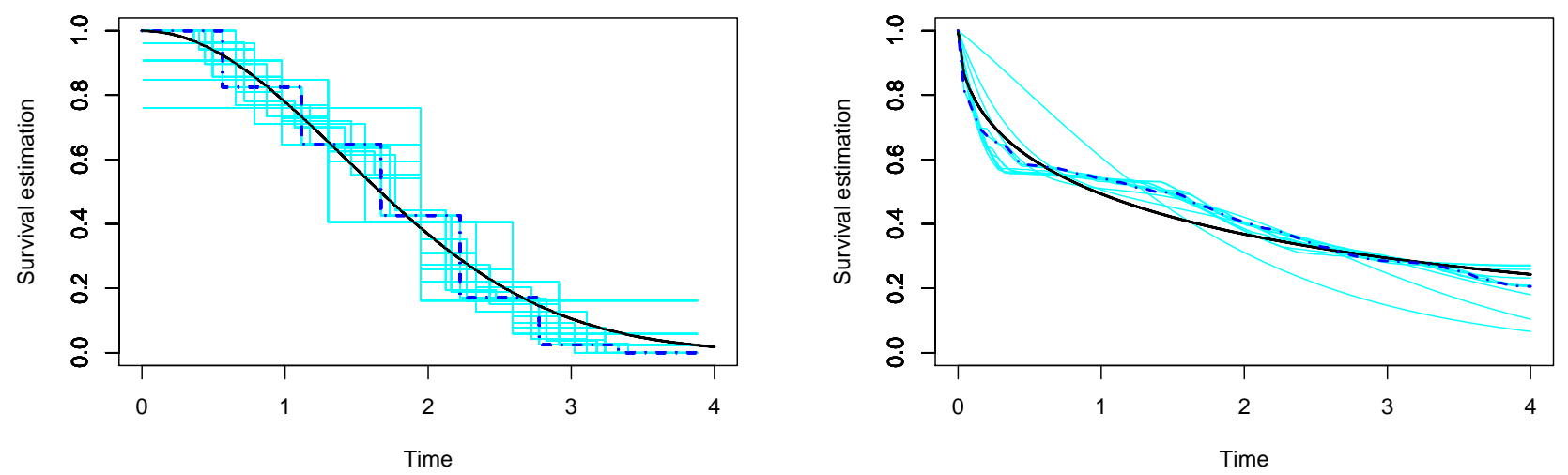

Figure 2. Model selection for mean squares estimators : Collection of estimators $\widehat{S}_{m}^{(Z)}$ for $m=1, \cdots, 12$ (cyan plain line) and selected estimator (blue dotdashed line) for a sample of size $n=300$ with histogram basis (left) for Model 1, scenario 1 and with Laguerre basis (right) for Model 2, scenario 2. True survival curve $S_{I}$ (black solid line).

most reliable. This fact is also illustrated on Figure 3 with bundles of estimators for Model 2 and 4. The boxplots on Figure 5 and 6 also confirm this fact. Except a small number of extreme error values (which means that model selection failed), the Least Squares estimator appears to be a quite good compromise for any distribution type of the event time and for any support of the inspection times.

Remark 3. A drawback of our estimation procedure is that it doesn't build a strict estimator of a survival function. In fact, the penalized estimator may start at a value different of 1 and may fail to be monotone. As it is consistent, this does not happen for large enough sample sizes. However, we propose an a posteriori transformation to correct these two facts. We compute first the original penalized estimator $\widehat{S}_{\hat{m}}$. Then, we reevaluate the coefficients of our penalized estimator by adding a constraint in the least squares contrast to make the estimator be equal to 1 at the origin. The constrained least squares contrast can be expressed with the Lagrange multiplier $\gamma_{n}(t)-\lambda(t(0)-1)$. From a computational point of view, the procedure is straightforward and leads to a smooth correction of the estimator. We do not investigate the theoretical properties of the resulting constrained estimator, but a study of its properties can be found in another context in Comte and Dion (2017). Finally, the procedure of Chernozhukov et al. (2009) available in the R-package Rearrangement allows to overcome the possible problem of monotony and can be applied without degrading the rate of the original estimator. These corrections are applied to the estimators plotted in Figures 1-4.

\section{Application to a real dataset}

In this section we study a dataset from Melbye et al. (1984). In this dataset, a cohort of homosexual men from two cities in Denmark has been examined for HIV-antibody positivity on six different dates: December 1981, April 1982, February 1983, September 1984, April 1987, and May 1989. The dataset comprises a total of 297 people who have been tested at least once. 

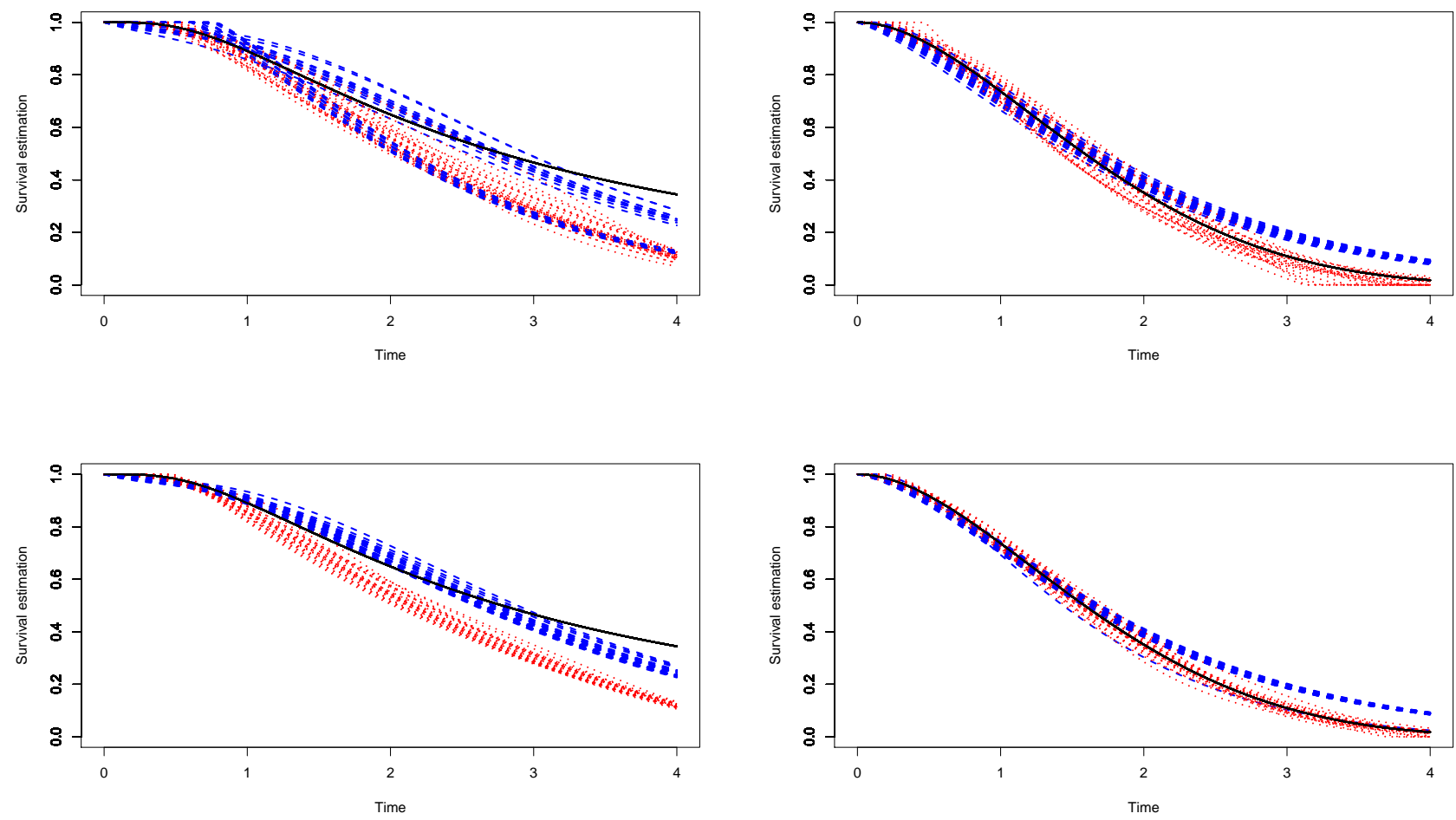

Figure 3. Bundles of 25 estimators : Anderson-Bergman and Yu estimators (red dotted) and Laguerre basis estimators (blue dashed), Model 3 (Left) and Model 4 (Right) for $n=300$ at the top and $n=1000$ at the bottom, all in scenario 2 .
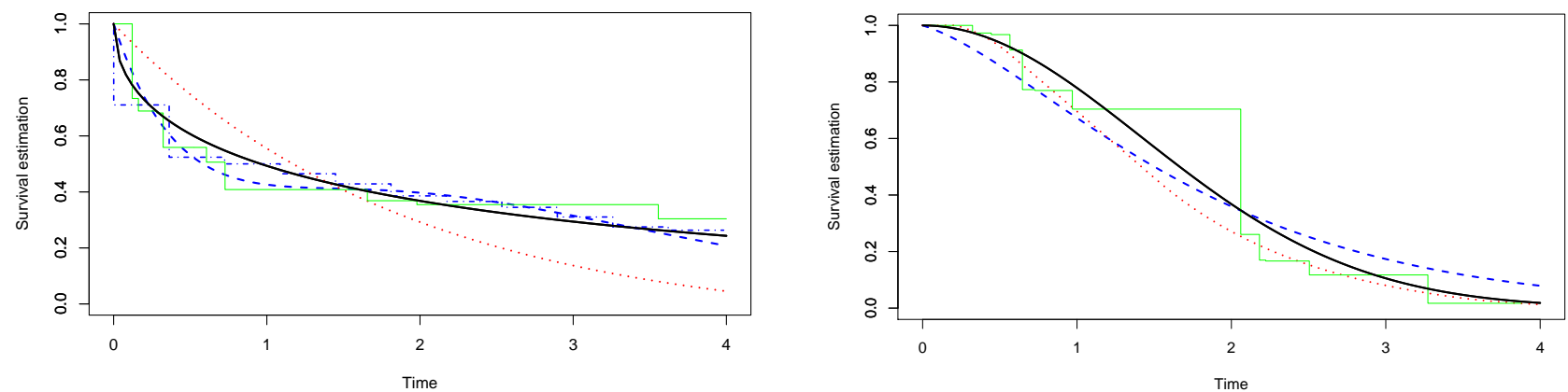

Figure 4. True survival function $S_{I}$ on $I=[0,4]$ (black solid line), AndersonBergman and $\mathrm{Yu}$ estimator (red dotted), our least squares estimators with Laguerre basis (blue dashed) and histogram basis (blue dotdashed) and NPMLE estimator (green step line) for Model 2 in scenario 1 on the left and Model 1 in scenario 4 on the right. 

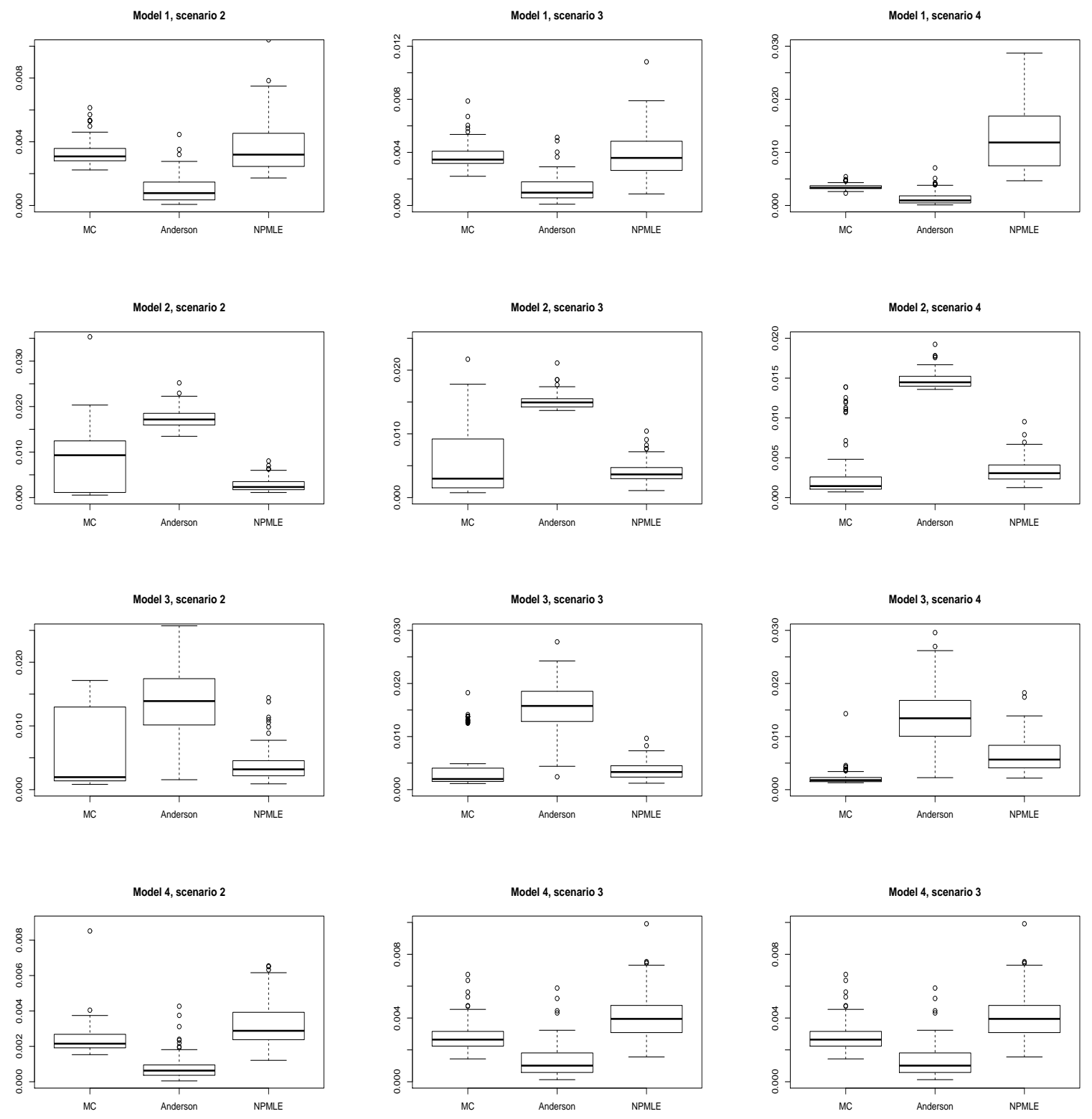

Figure 5. Average Mean Squared Error for sample size $n=300$. From top to bottom Model 1 to 4, from left to right Scenario 2 to 4 .

Among all these people, 26 were diagnosed with infection at the first examination date (which corresponds to $\delta_{i}=-1$ ), 39 were diagnosed with infection at another examination date (which corresponds to $\delta_{i}=0$ ) and 232 were examined without HIV infection (which corresponds to $\delta_{i}=1$ ). See also Becker and Melbye (1991) and Carstensen (1996) for more informations on the dataset.

Our new estimator with Laguerre basis is applied to the dataset using calendar time as the time scale. In order to deal with the high time values of the dataset which may cause numerical difficulties, we rescale the observations for the estimator computations. The rescaled sample 

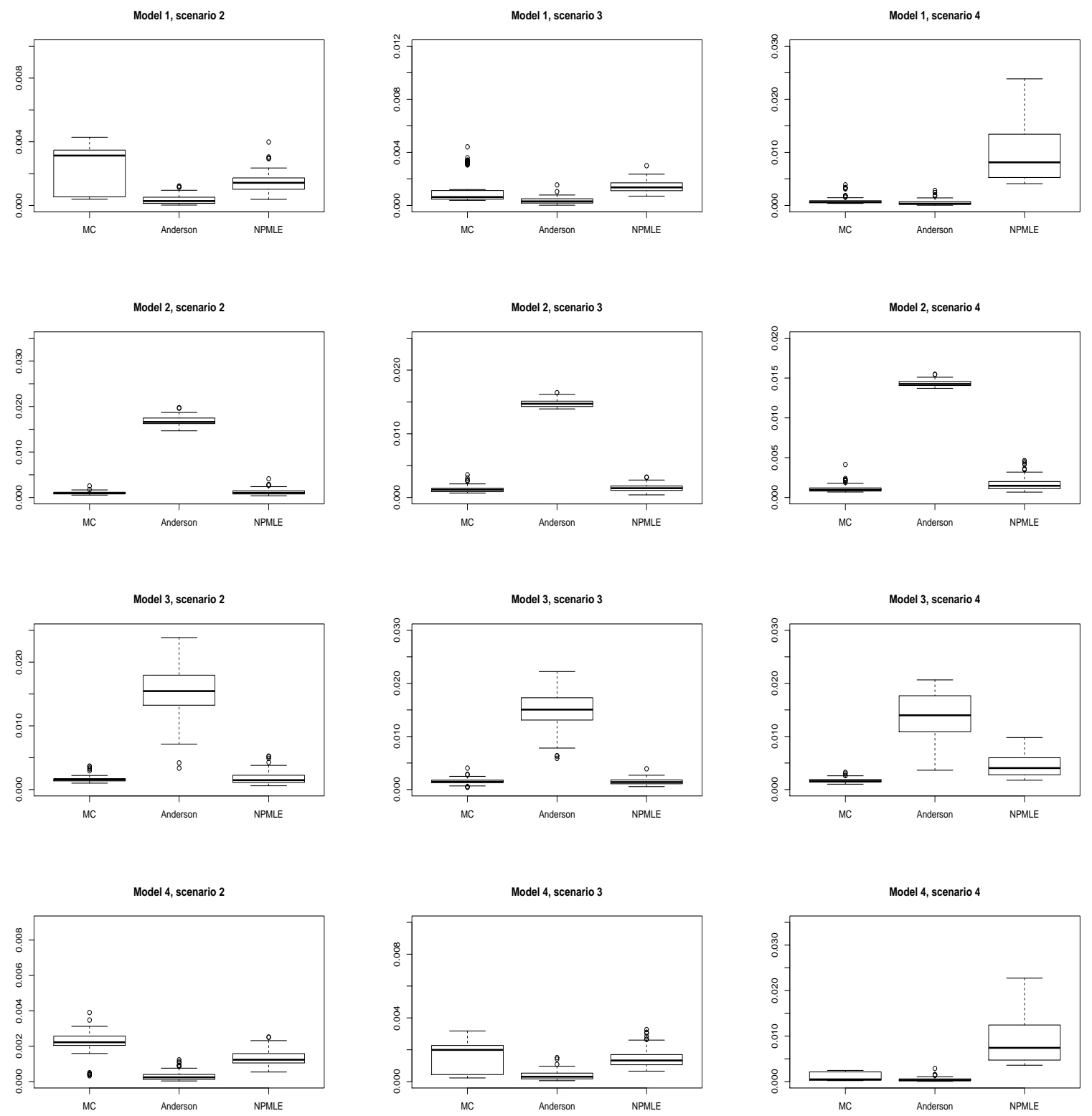

Figure 6. Average Mean Squared Error for sample size $n=1000$. From top to bottom Model 1 to 4, from left to right Scenario 2 to 4 .

$\left(L_{i}^{\prime}, U_{i}^{\prime}\right)_{1 \leq i \leq n}$ is obtained by applying the transformation $t \mapsto\left(t-\min \left(L_{i}\right)\right) /\left(\max \left(U_{i}\right)-\min \left(L_{i}\right)\right.$ to the original data $\left(L_{i}, U_{i}\right)_{1 \leq i \leq n}$. Then, the final curve is plotted in its original scale.

From the collection of models defined in (15), only four different models are allowed. Plots of the different estimators for each of these models are presented in Figure 7. Setting $\kappa=4$ as in the simulation studies, our selection procedure chooses the model $m=2$. As an alternative procedure to choose the correct model, we also used the R Capushe package based on slope heuristics (see Baudry et al., 2012): both the data-driven slope estimation and the dimension jump algorithms lead to the same model selection $(m=2)$. The corresponding estimator is displayed in Figure 
8 along with two competitors: the NPMLE implemented from the prodlim package and the Anderson-Bergman and $\mathrm{Yu}$ estimator implemented from the logconPH package. As described in Remark 3, the constrained version of our estimator is also displayed in Figure 8. Since 26 patients were diagnosed with infection at the first examination date, we chose to interpret the unconstrained version of our estimator. This estimator seems to be in accordance with the NPMLE, while providing a smoothed estimation of the survival function. On the other hand the Anderson-Bergman and $\mathrm{Yu}$ estimator provides very different survival estimates: for instance, it estimates to $35.4 \%$ the chance of being HIV negative among Danish homosexual men in 1986 and to $2.7 \%$ the chance of being HIV negative in 1990. On the contrary, the NPMLE and our estimator respectively estimate to $78.1 \%$ and $79.1 \%$ the chances of being HIV negative in 1986 and to $71.7 \%$ and $64.1 \%$ the chances of being HIV negative in 1990 . It seems that the Anderson-Bergman and $\mathrm{Yu}$ method is not adapted to this dataset because it implicitly assumes that the time distribution is log-concave while our estimator works for more general survival distributions. As a result, our method provides much more realistic survival estimates than their method.

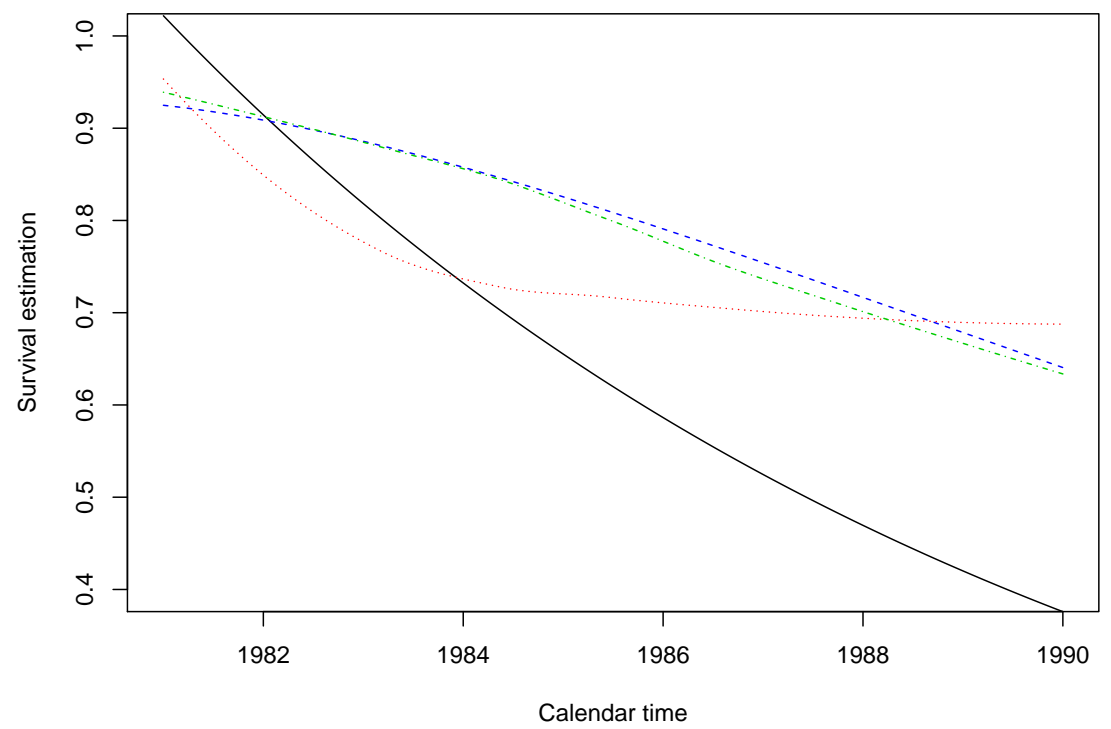

FiguRE 7. Our survival estimate of HIV infection using Laguerre basis. Black solid line corresponds to $m=1$, blue dashed line to $m=2$, red dotted to $m=3$ and green dotdash to $m=4$. Our selection procedure chooses the model $m=2$.

\section{ProOFs}

6.1. Proof of Proposition 1. Let $\Pi_{m}^{(L)}$ denote the orthogonal projection (for the scalar product in $\mathbb{R}^{n}$ ) on the subspace $\left\{{ }^{t}\left(t\left(L_{1}\right), \ldots, t\left(L_{n}\right)\right), t \in \Sigma_{m}\right\}$ of $\mathbb{R}^{n}$ and let $\Pi_{m}^{(L)} S$ be the projection of ${ }^{t}\left(S\left(L_{1}\right), \ldots, S\left(L_{n}\right)\right)$. Then by Pythagoras,

$\left\|\hat{S}_{m}^{(L)}-S_{I_{L}}\right\|_{n, L}^{2}=\left\|\Pi_{m}^{(L)} S-S_{I_{L}}\right\|_{n, L}^{2}+\left\|\hat{S}_{m}^{(L)}-\Pi_{m}^{(L)} S\right\|_{n, L}^{2}=\inf _{t \in \Sigma_{m}\left(I_{L}\right)}\left\|t-S_{I_{L}}\right\|_{n, L}^{2}+\left\|\hat{S}_{m}^{(L)}-\Pi_{m}^{(L)} S\right\|_{n, L}^{2}$. 


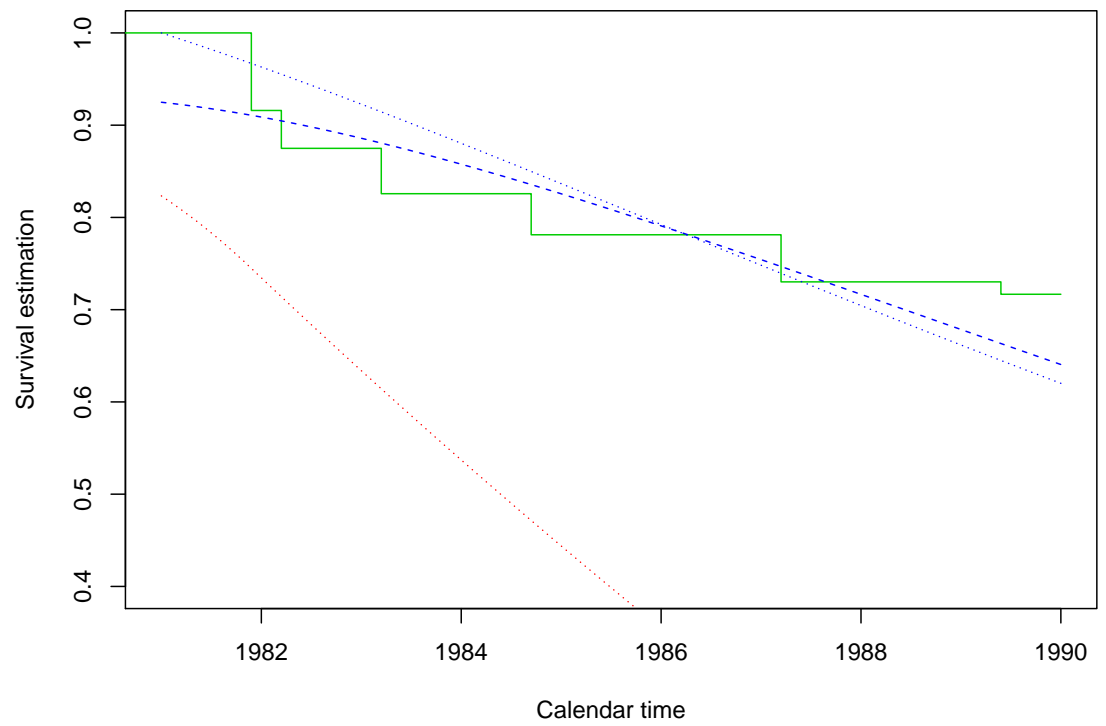

FIgURE 8. Survival estimates of HIV infection using the NPMLE (green solid line), our estimator with Laguerre basis after model selection with $m=2$ (blue dashed line for the original estimator and blue dotted for the constrained estimator) and the log-concave estimator from Anderson-Bergman and Yu (red dotted line).

By taking the expectation of the above formula, we have

$$
\mathbb{E}\left[\left\|\hat{S}_{m}^{(L)}-S_{I_{L}}\right\|_{n, L}^{2}\right] \leq \int_{t \in \Sigma_{m}\left(I_{L}\right)}\left\|t-S_{I_{L}}\right\|_{L}^{2}+\mathbb{E}\left[\left\|\hat{S}_{m}^{(L)}-\Pi_{m}^{(L)} S\right\|_{n, L}^{2}\right] .
$$

Now, we compute and bound $\mathbb{E}\left[\left\|\hat{S}_{m}^{(L)}-\Pi_{m}^{(L)} S\right\|_{n, L}^{2}\right]$. We have

$$
\overrightarrow{\widehat{S}}_{m}^{(L)}:=\left(\begin{array}{c}
\widehat{S}_{m}^{(L)}\left(L_{1}\right) \\
\vdots \\
\widehat{S}_{m}^{(L)}\left(L_{n}\right)
\end{array}\right)=\Phi_{m}^{(L)} \overrightarrow{\hat{a}}_{m}^{(L)}=\Phi_{m}^{(L)}\left({ }^{t} \Phi_{m}^{(L)} \Phi_{m}^{(L)}\right)^{-1}{ }^{t} \Phi_{m}^{(L)} \vec{\delta}^{(L)}
$$

We set $\Xi_{m}^{(L)}=\Phi_{m}^{(L)}\left({ }^{t} \Phi_{m}^{(L)} \Phi_{m}^{(L)}\right)^{-1}{ }^{t} \Phi_{m}^{(L)}$ and note that is corresponds to the matrix of the orthogonal projection $\Pi_{m}^{(L)}$. Therefore

$$
\Pi_{m}^{(L)} S=\Xi_{m}^{(L)} \mathbf{S}(L) \text { where } \mathbf{S}(L)={ }^{t}\left(S\left(L_{1}\right), \ldots, S\left(L_{n}\right)\right) .
$$

Therefore, denoting by $\vec{\varepsilon}^{(L)}(L)={ }^{t}\left(\varepsilon^{(L)}\left(L_{1}\right), \ldots, \varepsilon^{(L)}\left(L_{n}\right)\right)$, where $\varepsilon^{(L)}\left(L_{i}\right)=1-\mathbb{1}_{\delta_{i}=-1}-S\left(L_{i}\right)$, we get

$$
\left\|\hat{S}_{m}^{(L)}-\Pi_{m}^{(L)} S\right\|_{n, L}^{2}=\left\|\Xi_{m}^{(L)} \vec{\varepsilon}^{(L)}(L)\right\|_{n, L}^{2}=\frac{1}{n}{ }^{t} \vec{\varepsilon}^{(L)}(L){ }^{t} \Xi_{m}^{(L)} \Xi_{m}^{(L)} \vec{\varepsilon}^{(L)}(L)=\frac{1}{n}{ }^{t} \vec{\varepsilon}^{(L)}(L) \Xi_{m}^{(L)} \vec{\varepsilon}^{(L)}(L) .
$$


Now,

$$
\begin{aligned}
\mathbb{E}\left[\vec{\varepsilon}^{(L)}(L) \Xi_{m}^{(L)} \vec{\varepsilon}^{(L)}(L)\right] & =\sum_{1 \leq i, k \leq n} \mathbb{E}\left(\varepsilon^{(L)}\left(L_{i}\right) \varepsilon^{(L)}\left(L_{k}\right)\left[\Xi_{m}^{(L)}\right]_{i, k}\right)=\sum_{i=1}^{n} \mathbb{E}\left(\varepsilon^{(L)}\left(L_{i}\right)^{2}\left[\Xi_{m}^{(L)}\right]_{i, i}\right) \\
& =\sum_{i=1}^{n} \mathbb{E}\left(S\left(L_{i}\right)\left(1-S\left(L_{i}\right)\right)\left[\Xi_{m}^{(L)}\right]_{i, i}\right) \\
& \leq \frac{1}{4} \sum_{i=1}^{n} \mathbb{E}\left(\left[\Xi_{m}^{(L)}\right]_{i, i}\right)=\frac{1}{4} \mathbb{E}\left(\operatorname{Tr}\left(\Xi_{m}^{(L)}\right)\right) .
\end{aligned}
$$

Indeed $\Xi_{m}^{(L)}$ is a symmetric positive matrix, so that ${ }^{t} x \Xi_{m}^{(L)} x>0$ for all vector $x$, and thus its diagonal coefficients are positive. Now $\operatorname{Tr}\left(\Xi_{m}^{(L)}\right)=\operatorname{Tr}\left(\left({ }^{t} \Phi_{m}^{(L)} \Phi_{m}^{(L)}\right)^{-1}{ }^{t} \Phi_{m}^{(L)} \Phi_{m}^{(L)}\right)=\operatorname{Tr}\left(I_{m}\right)=m$. Thus

$$
\mathbb{E}\left[\left\|\hat{S}_{m}^{(L)}-\Pi_{m}^{(L)} S\right\|_{n, L}^{2}\right] \leq \frac{1}{4} \frac{m}{n}
$$

and plugging this in (19) gives the result of Proposition 1 for $\hat{S}_{m}^{(L)}$. The same ideas give the result for $\hat{S}_{m}^{(U)}$.

6.2. Proof of Proposition 3. We start by the contrast decomposition: let $t, t^{\prime} \in \Sigma_{m}$, then

$$
\begin{aligned}
\gamma_{n}(t)-\gamma_{n}\left(t^{\prime}\right)= & \left\|t-S_{I}\right\|_{n, U}^{2}+\left\|t-S_{I}\right\|_{n, L}^{2}-\left(\left\|t^{\prime}-S_{I}\right\|_{n, U}^{2}+\left\|t^{\prime}-S_{I}\right\|_{n, L}^{2}\right) \\
& -2 \nu_{n, U}\left(t-t^{\prime}\right)-2 \nu_{n, L}\left(t-t^{\prime}\right),
\end{aligned}
$$

where

$$
\nu_{n, U}(t)=\frac{1}{n} \sum_{i=1}^{n} t\left(U_{i}\right)\left(\mathbb{1}_{\delta_{i}=1}-S\left(U_{i}\right)\right), \quad \nu_{n, L}(t)=\frac{1}{n} \sum_{i=1}^{n} t\left(L_{i}\right)\left(\mathbb{1}_{\delta_{i} \neq-1}-S\left(L_{i}\right)\right) .
$$

Writing that $\gamma_{n}\left(\widehat{S}_{m}\right) \leq \gamma_{n}\left(S_{m}\right)$ for any $S_{m} \in \Sigma_{m}$, we get $\left\|\widehat{S}_{m}-S_{I}\right\|_{n, U}^{2}+\left\|\widehat{S}_{m}-S_{I}\right\|_{n, L}^{2} \leq\left\|S_{m}-S_{I}\right\|_{n, U}^{2}+\left\|S_{m}-S_{I}\right\|_{n, L}^{2}+2 \nu_{n, U}\left(\widehat{S}_{m}-S_{m}\right)+2 \nu_{n, L}\left(\widehat{S}_{m}-S_{m}\right)$.

Denoting by $\varepsilon^{(L)}\left(L_{i}\right)=\mathbb{1}_{\delta_{i} \neq-1}-S\left(L_{i}\right)$ and $\varepsilon^{(U)}\left(U_{i}\right)=\mathbb{1}_{\delta_{i}=1}-S\left(U_{i}\right)$, the inequality writes

$$
\begin{aligned}
\mathbb{E}\left[\left\|\widehat{S}_{m}-S_{I}\right\|_{n, U}^{2}+\left\|\widehat{S}_{m}-S_{I}\right\|_{n, L}^{2}\right] \leq \mathbb{E}\left[\left\|S_{m}-S_{I}\right\|_{n, U}^{2}+\left\|S_{m}-S_{I}\right\|_{n, L}^{2}\right] \\
+\frac{2}{n} \mathbb{E}\left[\sum_{i=1}^{n}\left(\varepsilon^{(L)}\left(L_{i}\right)\left(\widehat{S}_{m}-S_{m}\right)\left(L_{i}\right)+\varepsilon^{(U)}\left(U_{i}\right)\left(\widehat{S}_{m}-S_{m}\right)\left(U_{i}\right)\right)\right] \\
\quad \leq\left\|S_{m}-S_{I}\right\|_{U}^{2}+\left\|S_{m}-S_{I}\right\|_{L}^{2} \\
+\frac{2}{n} \mathbb{E} \underbrace{\left[\sum_{i=1}^{n}\left(\varepsilon^{(L)}\left(L_{i}\right) \widehat{S}_{m}\left(L_{i}\right)+\varepsilon^{(U)}\left(U_{i}\right) \widehat{S}_{m}\left(U_{i}\right)\right)\right]}_{:=\mathbb{T}}
\end{aligned}
$$

Let us set

$$
\Theta_{m}={ }^{t} \Phi_{m}^{(L)} \Phi_{m}^{(L)}+{ }^{t} \Phi_{m}^{(U)} \Phi_{m}^{(U)}
$$

As we have

we find

$$
\mathbb{T}=\left({ }^{t} \vec{\varepsilon}^{(L)}(L) \Phi_{m}^{(L)}+{ }^{t} \vec{\varepsilon}^{(U)}(U) \Phi_{m}^{(U)}\right) \Theta_{m}^{-1}\left({ }^{t} \Phi_{m}^{(L)} \vec{\delta}^{(L)}+{ }^{t} \Phi_{m}^{(U)} \vec{\delta}^{(U)}\right)
$$

$$
\mathbb{E}(\mathbb{T})=\mathbb{E}\left(\left({ }^{t} \bar{\varepsilon}^{(L)}(L) \Phi_{m}^{(L)}+{ }^{t} \vec{\varepsilon}^{(U)}(U) \Phi_{m}^{(U)}\right) \Theta_{m}^{-1}\left({ }^{t} \Phi_{m}^{(L)} \vec{\varepsilon}^{(L)}(L)+{ }^{t} \Phi_{m}^{(U)} \vec{\varepsilon}^{-(U)}(U)\right)\right) .
$$


We get

$$
\mathbb{E}(\mathbb{T}):=\mathbb{E}\left(\mathbb{T}_{L}\right)+\mathbb{E}\left(\mathbb{T}_{U}\right)+\mathbb{E}\left(\mathbb{T}_{L, U}\right)
$$

where, by using that $\mathbb{E}\left[\left(\varepsilon^{(L)}\left(L_{i}\right)\right)^{2} \mid L_{i}\right]=S\left(L_{i}\right)\left(1-S\left(L_{i}\right)\right)$ and $\mathbb{E}\left[\left(\varepsilon^{(U)}\left(U_{i}\right)\right)^{2} \mid U_{i}\right]=S\left(U_{i}\right)(1-$ $S\left(U_{i}\right)$,

$$
\mathbb{T}_{L}=\sum_{i=1}^{n} S\left(L_{i}\right)\left(1-S\left(L_{i}\right)\right)\left[\Phi_{m}^{(L)} \Theta_{m}^{-1}{ }^{t} \Phi_{m}^{(L)}\right]_{i, i}, \mathbb{T}_{U}=\sum_{i=1}^{n} S\left(U_{i}\right)\left(1-S\left(U_{i}\right)\right)\left[\Phi_{m}^{(U)} \Theta_{m}^{-1}{ }^{t} \Phi_{m}^{(U)}\right]_{i, i}
$$

and

$$
\mathbb{T}_{L, U}=\sum_{i=1}^{n} S\left(U_{i}\right)\left(1-S\left(L_{i}\right)\right)\left[\Phi_{m}^{(L)} \Theta_{m}^{-1}{ }^{t} \Phi_{m}^{(U)}+\Phi_{m}^{(U)} \Theta_{m}^{-1}{ }^{(L)} \Phi_{m}^{(L)}\right]_{i, i}
$$

Let us denote by $\|\vec{x}\|_{2, d}^{2}=x_{1}^{2}+\cdots+x_{d}^{2}$ the euclidean norm of a vector $\vec{x}$ of $\mathbb{R}^{d}$ and by $\vec{e}_{i, d}$ the $i$-th canonical basis vector in $\mathbb{R}^{d}$, that is the $d$-dimensional vector with all coordinates null except the $i$-th which is equal to 1 . Then we have, for $Z=L, U$,

$$
\left[\Phi_{m}^{(Z)} \Theta_{m}^{-1}{ }^{t} \Phi_{m}^{(Z)}\right]_{i, i}={ }^{t} \vec{e}_{i, n} \Phi_{m}^{(Z)} \Theta_{m}^{-1}{ }^{t} \Phi_{m}^{(Z)} \vec{e}_{i, n}=\left\|\Theta_{m}^{-1 / 2}{ }^{t} \Phi_{m}^{(Z)} \vec{e}_{i, n}\right\|_{2, n}^{2} \geq 0
$$

where $\Theta_{m}^{-1 / 2}$ is a matrix symmetric square root of $\Theta_{m}^{-1}$. Thus for $Z=L, U$, we have

$$
\mathbb{E}\left(\mathbb{T}_{Z}\right) \leq \frac{1}{4} \mathbb{E}\left(\sum_{i=1}^{n}\left[\Phi_{m}^{(Z)} \Theta_{m}^{-1}{ }^{t} \Phi_{m}^{(Z)}\right]_{i, i}\right)=\frac{1}{4} \mathbb{E}\left(\operatorname{Tr}\left(\Phi_{m}^{(Z)} \Theta_{m}^{-1}{ }^{t} \Phi_{m}^{(Z)}\right)\right)=\frac{1}{4} \mathbb{E}\left(\operatorname{Tr}\left(\Theta_{m}^{-1}{ }^{t} \Phi_{m}^{(Z)} \Phi_{m}^{(Z)}\right)\right) .
$$

It follows that

$$
\mathbb{E}\left(\mathbb{T}_{L}+\mathbb{T}_{U}\right) \leq \frac{1}{4} \mathbb{E}\left(\operatorname{Tr}\left(\Theta_{m}^{-1}\left({ }^{t} \Phi_{m}^{(L)} \Phi_{m}^{(L)}+{ }^{t} \Phi_{m}^{(U)} \Phi_{m}^{(U)}\right)\right)\right)=\frac{1}{4} \operatorname{Tr}\left(I_{m}\right)=\frac{m}{4} .
$$

Now we prove that $\mathbb{T}_{L, U} \leq m$. Let us set $D^{2}=\operatorname{diag}\left(d_{1}^{2}, \ldots, d_{n}^{2}\right)$ with $d_{i}^{2}=S\left(U_{i}\right)\left(1-S\left(L_{i}\right)\right)$. We have

$$
\mathbb{T}_{L, U}=\operatorname{Tr}\left(D^{2}\left(\Phi_{m}^{(L)} \Theta_{m}^{-1}{ }^{t} \Phi_{m}^{(U)}+\Phi_{m}^{(U)} \Theta_{m}^{-1}{ }^{t} \Phi_{m}^{(L)}\right)\right)=\operatorname{Tr}\left(\Theta_{m}^{-1}\left({ }^{t} \Phi_{m}^{(U)} D^{2} \Phi_{m}^{(L)}+{ }^{t} \Phi_{m}^{(L)} D^{2} \Phi_{m}^{(U)}\right)\right) .
$$

Let us denote by $\Theta_{m, D}:={ }^{t} \Phi_{m}^{(L)} D^{2} \Phi_{m}^{(L)}+{ }^{t} \Phi_{m}^{(U)} D^{2} \Phi_{m}^{(U)}$. We remark that, for any vector $\vec{x} \in \mathbb{R}^{m}$, we have

$$
{ }^{t} \vec{x}^{t}\left(D ( \Phi _ { m } ^ { ( L ) } - \Phi _ { m } ^ { ( U ) } ) \left(D\left(\Phi_{m}^{(L)}-\Phi_{m}^{(U)}\right) \vec{x}=\left\|D\left(\Phi_{m}^{(L)}-\Phi_{m}^{(U)}\right) \vec{x}\right\|_{2, n}^{2} \geq 0\right.\right.
$$

and the term is also equal to

$$
{ }^{t} \vec{x}^{t}\left(D\left(\Phi_{m}^{(L)}-\Phi_{m}^{(U)}\right)\right)\left(D\left(\Phi_{m}^{(L)}-\Phi_{m}^{(U)}\right)\right) \vec{x}={ }^{t} \vec{x}^{t}\left(\Theta_{m, D}-\left({ }^{t} \Phi_{m}^{(U)} D^{2} \Phi_{m}^{(L)}+{ }^{t} \Phi_{m}^{(L)} D^{2} \Phi_{m}^{(U)}\right)\right) \vec{x} .
$$

Setting $\vec{x}=\Theta_{m}^{-1 / 2} \vec{y}$, we get

$$
{ }^{t} \vec{y} \Theta_{m}^{-1 / 2}\left({ }^{t} \Phi_{m}^{(U)} D^{2} \Phi_{m}^{(L)}+{ }^{t} \Phi_{m}^{(L)} D^{2} \Phi_{m}^{(U)}\right) \Theta_{m}^{-1 / 2} \vec{y} \leq{ }^{t} \vec{y} \Theta_{m}^{-1 / 2} \Theta_{m, D} \Theta_{m}^{-1 / 2} \vec{y} .
$$

Choosing $\vec{y}=\vec{e}_{i, m}$ and summing up the terms over $i$, we obtain that

$$
\begin{aligned}
\operatorname{Tr}\left(\Theta_{m}^{-1}\left({ }^{t} \Phi_{m}^{(U)} D^{2} \Phi_{m}^{(L)}+{ }^{t} \Phi_{m}^{(L)} D^{2} \Phi_{m}^{(U)}\right)\right) & =\operatorname{Tr}\left(\Theta_{m}^{-1 / 2}\left({ }^{t} \Phi_{m}^{(U)} D^{2} \Phi_{m}^{(L)}+{ }^{t} \Phi_{m}^{(L)} D^{2} \Phi_{m}^{(U)}\right) \Theta_{m}^{-1 / 2}\right) \\
& \leq \operatorname{Tr}\left(\Theta_{m}^{-1 / 2} \Theta_{m, D} \Theta_{m}^{-1 / 2}\right)=\operatorname{Tr}\left(\Theta_{m, D} \Theta_{m}^{-1}\right) .
\end{aligned}
$$

Now, let $\lambda$ be an eigenvalue of $\Theta_{m, D} \Theta_{m}^{-1}$, associated to a nonzero eigenvector $\vec{x}, \vec{x} \in \mathbb{R}^{m}$, we have

$$
\Theta_{m}^{-1} \Theta_{m, D} \vec{x}=\lambda \vec{x} \Rightarrow \Theta_{m, D} \vec{x}=\lambda \Theta_{m} \vec{x} \Rightarrow{ }^{t} \vec{x} \Theta_{m, D} \vec{x}=\lambda{ }^{t} \vec{x} \Theta_{m} \vec{x} .
$$


It is easy to see that ${ }^{t} \vec{x} \Theta_{m, D} \vec{x} \geq 0$ and ${ }^{t} \vec{x} \Theta_{m} \vec{x}>0$ as $\Theta_{m}$ is assumed to be invertible, and thus

$$
\lambda=\frac{{ }^{t} \vec{x} \Theta_{m, D} \vec{x}}{{ }^{t} \Theta_{m} \vec{x}}=\frac{{ }^{t} \vec{z}_{1} D^{2} \vec{z}_{1}+{ }^{t} \vec{z}_{2} D^{2} \vec{z}_{2}}{{ }^{t} \vec{z}_{1} \vec{z}_{1}+{ }^{t} \vec{z}_{2} \vec{z}_{2}}
$$

where $\vec{z}_{k}=\Phi_{m}^{(Z)} \vec{x} \in \mathbb{R}^{n}$ where $k=1$ for $Z=L$ and $k=2$ for $Z=U$. It follows that

$$
\lambda=\frac{\sum_{i=1}^{n} d_{i}^{2}\left(\left[\vec{z}_{1}\right]_{i}^{2}+\left[\vec{z}_{2}\right]_{i}^{2}\right)}{\sum_{i=1}^{n}\left(\left[\vec{z}_{1}\right]_{i}^{2}+\left[\vec{z}_{2}\right]_{i}^{2}\right)} \leq 1
$$

since $\forall i, d_{i}^{2} \leq 1$. Now the trace of a square $m \times m$ matrix which has all its eigenvalues less than 1 (and is diagonalizable), is less than $m$. This implies that

$$
\mathbb{T}_{L, U} \leq m \text {. }
$$

Gathering (23), (24) and (25), we get that

$$
\frac{2}{n} \mathbb{E}(\mathbb{T}) \leq \frac{5}{2} \frac{m}{n}
$$

and plugging this in (21), we obtain, for any $S_{m} \in \Sigma_{m}$,

$$
\mathbb{E}\left[\left\|\widehat{S}_{m}-S_{I}\right\|_{n, U}^{2}+\left\|\widehat{S}_{m}-S_{I}\right\|_{n, L}^{2}\right] \leq \mathbb{E}\left[\left\|S_{m}-S_{I}\right\|_{n, U}^{2}+\left\|S_{m}-S_{I}\right\|_{n, L}^{2}\right]+\frac{5}{2} \frac{m}{n} .
$$

Now, using that $\mathbb{E}\left[\left\|S_{m}-S_{I}\right\|_{n, Z}^{2}\right]=\left\|S_{m}-S_{I}\right\|_{Z}^{2}$ for $Z=L, U$, we obtain the result of Proposition 3 .

6.3. Complement about histogram. In fact, Birgé's proposal also involve the following cardinalities

$$
\begin{gathered}
Q_{j, k}:=\operatorname{Card}\left\{i \in\{1, \ldots, n\}, L_{i} \in I_{j} \text { and } U_{i} \in I_{k}\right\}, \\
Q_{j, k}^{\prime}=\operatorname{Card}\left\{i \in\{1, \ldots, n\}, L_{i} \in I_{j}, U_{i} \in I_{k} \text { and } \delta_{i}=0\right\} .
\end{gathered}
$$

The estimator defined with $\tilde{\gamma}_{n}$ would involve such coefficients. Indeed, it relies on the inversion of a matrix with diagonal coefficients equal to $(m / n)\left(M_{j}+N_{j}-2 Q_{j, j}\right)$ and non diagonal terms equal to $-(m / n) Q_{j, k}$. In other words, a matrix equal to

$$
\widetilde{\Psi}_{m}=(m / n)\left(\operatorname{Diag}\left(M_{1}+N_{1}, \ldots, M_{m}+N_{m}\right)+\mathbf{Q}\right),
$$

where $\mathbf{Q}=\left(Q_{j, k}\right)_{1 \leq j, k \leq m}$. Note that $\mathbf{Q}$ is triangular as $Q_{j, k}=0$ if $j>k$ and thus $\widetilde{\Psi}_{m}$ is triangular. Therefore, it is invertible as soon as its diagonal coefficients are non zero. The computation of the "difference estimator" would also involve all the specific coefficients defined in Birgé (1999). More precisely, if we denote by $Q_{j, \bullet}^{\prime}:=\sum_{k} Q_{j, k}^{\prime}=\sum_{k=j}^{m} Q_{j, k}^{\prime}$ and $Q_{\bullet, k}^{\prime}:=$ $\sum_{j} Q_{j, k}^{\prime}=\sum_{j=1}^{k} Q_{j, k}^{\prime}$, the "difference estimator" would lead to compute $\left[\Psi_{m}^{(3)}\right]^{-1}(\sqrt{m} / n)\left(Q_{j, \bullet}^{\prime}-\right.$ $\left.Q_{\bullet}^{\prime}, j\right)_{1 \leq j \leq m}$. But the link is not clear and Birgé's proposal includes adaptation while we would propose a second step.

6.4. Proof of Theorem 1. The result is mainly a particular case of Theorem 2.1 of Comte and Genon-Catalot (2018), in a simpler case of bounded noise. This is why we only present here a sketch of proof.

The main tools in the proof of Comte and Genon-Catalot (2018) are the Talagrand Inequality and Tropp's (2015) matricial Bernstein Inequality. Both still apply here. For Talagrand, we loose the independence property of the noise, but get a simplified setting due to the boudedness property of $\varepsilon^{(L)}\left(L_{i}\right)=\mathbb{1}_{\delta_{i} \neq-1}-S\left(L_{i}\right)$ and $\varepsilon^{(U)}\left(U_{i}\right)=\mathbb{1}_{\delta_{i}=1}-S\left(U_{i}\right)$. For Tropp's Inequality, it allows to have here the following fundamental Lemma: 
Lemma 1. Let $\left(L_{1}, U_{1}\right), \ldots,\left(L_{n}, U_{n}\right)$ be i.i.d. such that the densities $f_{U}$ and $f_{L}$ are bounded, $\sup _{x \in I} f_{Z}(x):=\left\|f_{Z}\right\|_{\infty}<+\infty$ for $Z=L, U$. Let the basis be such that $\left\|\sum_{j=0}^{m-1} \varphi_{j}^{2}\right\|_{\infty} \leq c_{\varphi}^{2} m$. Then, for all $u>0$,

$$
\mathbb{P}\left[\left\|\Psi_{m}-\widehat{\Psi}_{m}\right\|_{\mathrm{op}} \geq u\right] \leq 2 m \exp \left(-\frac{n u^{2} / 2}{2 c_{\varphi}^{2}\left[\left(\left\|f_{L}\right\|_{\infty}+\left\|f_{U}\right\|_{\infty}\right)+u / 3\right]}\right) .
$$

The proof is the same as the proof of Proposition 2.2 in Comte and Genon-Catalot (2018) with here the bound $c_{\varphi}^{2} m / n$ in (26) replaced by $2 c_{\varphi}^{2} m / n$ and the bound on $\nu_{n}\left(\mathbf{S}_{m}\right), c_{\varphi}^{2}\|f\|_{\infty} m / n$ replaced by $2 c_{\varphi}^{2}\left(\left\|f_{L}\right\|_{\infty}+\left\|f_{U}\right\|_{\infty}\right) m / n$.

This result is useful to study the set $\Omega_{n}$ defined by

$$
\Omega_{n}=\cap_{m \in \mathcal{M}_{n}} \Omega_{m} \quad \text { with } \quad \Omega_{m}=\left\{\left|\frac{\|t\|_{n}^{2}}{\|t\|_{L+U}^{2}}-1\right| \leq \frac{1}{2}, \forall t \in \Sigma_{m}(I) \backslash\{0\}\right\} .
$$

where $\|t\|_{n}^{2}=\|t\|_{n, L}^{2}+\|t\|_{n, U}^{2}$. Indeed Lemma 7.2 in Comte and Genon-Catalot (2018) can be written here as follows:

Lemma 2. Under the assumptions of Theorem $1, \mathbb{P}\left(\Omega_{n}^{c}\right) \leq c / n^{4}$ where $c$ is a positive constant.

To understand the link between Lemma 1 and Lemma 2, we mention that the main point of the proof is the equality

$$
\begin{aligned}
& \mathbb{P}\left(\exists t \in \Sigma_{m}(I),\left|\frac{\|t\|_{n}^{2}}{\|t\|_{L+U}^{2}}-1\right| \leq \frac{1}{2}\right) \\
= & \mathbb{P}\left(\sup _{t \in \Sigma_{m}(I),\|t\|_{L+U}=1}\left|\frac{1}{n} \sum_{i=1}^{n}\left[t^{2}\left(L_{i}\right)+t^{2}\left(U_{i}\right)-\mathbb{E}\left(t^{2}\left(L_{i}\right)+t^{2}\left(U_{i}\right)\right)\right]\right|>\frac{1}{2}\right),
\end{aligned}
$$

and the bound

$$
\sup _{t \in \Sigma_{m}(I),\|t\|_{L+U}=1}\left|\frac{1}{n} \sum_{i=1}^{n}\left[t^{2}\left(L_{i}\right)+t^{2}\left(U_{i}\right)-\mathbb{E}\left(t^{2}\left(L_{i}\right)+t^{2}\left(U_{i}\right)\right)\right]\right| \leq\left\|\Psi_{m}^{-1}\right\|_{\mathrm{op}}\left\|\widehat{\Psi}_{m}-\Psi_{m}\right\|_{\mathrm{op}} .
$$

Let us start the proof of Theorem 1 in a simplified context: we consider the estimator $\widehat{S}_{m}$ with $\hat{m}$ selected in the non random collection $\mathcal{M}_{n}$ and the empirical norm for the risk. The step from this to the effective random collection is given in the proof of Theorem 2.1 of Comte and Genon-Catalot (2018) and the last step to get a risk bound in term of integral norm weighted by $f_{L}+f_{U}$ in Corollary 2.1 therein. The starting point is the contrast decomposition (20). We use this to write that, for all $m \in \mathcal{M}_{n}$, for all $S_{m} \in \Sigma_{m}(I)$ :

$$
\gamma_{n}\left(\widehat{S}_{\hat{m}}\right)+\operatorname{pen}(\hat{m}) \leq \gamma_{n}\left(S_{m}\right)+\operatorname{pen}(m) .
$$

We get

$$
\begin{aligned}
\left\|\widehat{S}_{\hat{m}}-S_{I}\right\|_{n, U}^{2}+\left\|\widehat{S}_{\hat{m}}-S_{I}\right\|_{n, L}^{2} \leq & \left\|S_{m}-S_{I}\right\|_{n, U}^{2}+\left\|S_{m}-S_{I}\right\|_{n, L}^{2}+\operatorname{pen}(m) \\
& +2 \nu_{n, U}\left(\widehat{S}_{\hat{m}}-S_{m}\right)+2 \nu_{n, L}\left(\widehat{S}_{\hat{m}}-S_{m}\right)-\operatorname{pen}(\hat{m}) .
\end{aligned}
$$

Define

$$
\nu_{n}(t)=\nu_{n, L}(t)+\nu_{n, U}(t)=\frac{1}{n} \sum_{i=1}^{n}\left[\varepsilon^{(L)}\left(L_{i}\right) t\left(L_{i}\right)+\varepsilon^{(U)}\left(U_{i}\right) t\left(U_{i}\right)\right]
$$

and recall that $\mathbb{E}\left(\|t\|_{n}^{2}\right)=\mathbb{E}\left(\|t\|_{n, L}^{2}\right)+\mathbb{E}\left(\|t\|_{n, U}^{2}\right)=\|t\|_{L+U}^{2}=\int t^{2}(x)\left(f_{L}(x)+f_{U}(x)\right) d x$. 
In the following, we write $\Sigma_{m}$ for $\Sigma_{m}(I)$, for sake of brevity. Taking expectation of (27) yields

$$
\begin{aligned}
\mathbb{E}\left(\left\|\widehat{S}_{\hat{m}}-S_{I}\right\|_{n}^{2}\right) \leq & \left\|S_{m}-S_{I}\right\|_{L+U}^{2}+\operatorname{pen}(m) \\
& +2 \mathbb{E}\left(\left\|\widehat{S}_{\hat{m}}-S_{m}\right\|_{L+U} \sup _{t \in \Sigma_{m}+\Sigma_{\hat{m}},\|t\|_{L+U}=1}\left|\nu_{n}(t)\right|\right)-\mathbb{E}(\operatorname{pen}(\hat{m})) \\
\leq & \left\|S_{m}-S_{I}\right\|_{L+U}^{2}+\operatorname{pen}(m)+\frac{1}{4} \mathbb{E}\left(\left\|\widehat{S}_{\hat{m}}-S_{m}\right\|_{L+U}^{2}\right) \\
& +4 \mathbb{E}\left(\sup _{t \in \Sigma_{m}+\Sigma_{\hat{m}},\|t\|_{L+U}=1} \nu_{n}^{2}(t)\right)-\mathbb{E}(\operatorname{pen}(\hat{m})),
\end{aligned}
$$

where we use that $2|a b| \leq(1 / 4) a^{2}+4 b^{2}$ for all real numbers $a, b$. Now we bound separately the terms on $\Omega_{n}$ and $\Omega_{n}^{c}$ where $\Omega_{n}$ is defined by (26). We get

$$
\begin{aligned}
\mathbb{E}\left(\left\|\widehat{S}_{\hat{m}}-S_{I}\right\|_{n}^{2} \mathbb{1}_{\Omega_{n}}\right) \leq & \left\|S_{m}-S_{I}\right\|_{L+U}^{2}+\operatorname{pen}(m)+\frac{1}{4} \mathbb{E}\left(\left\|\widehat{S}_{\hat{m}}-S_{m}\right\|_{n}^{2} \mathbb{1}_{\Omega_{n}}\right) \\
& +4 \mathbb{E}\left(\sup _{t \in \Sigma_{m}+\Sigma_{\hat{m}},\|t\|_{L+U}=1} \nu_{n}^{2}(t)\right)-\mathbb{E}(\operatorname{pen}(\hat{m})) .
\end{aligned}
$$

Thus

$$
\begin{aligned}
\frac{1}{2} \mathbb{E}\left(\left\|\widehat{S}_{\hat{m}}-S_{I}\right\|_{n}^{2} \mathbb{1}_{\Omega_{n}}\right) \leq & \frac{3}{2}\left\|S_{m}-S_{I}\right\|_{L+U}^{2}+\operatorname{pen}(m) \\
& +4 \mathbb{E}\left(\sup _{t \in \Sigma_{m}+\Sigma_{\hat{m}},\|t\|_{L+U}=1} \nu_{n}^{2}(t)-p(m, \hat{m})\right)_{+}+\mathbb{E}(4 p(m, \hat{m})-\operatorname{pen}(\hat{m}))
\end{aligned}
$$

Then we can apply Talagrand inequality to get the Lemma:

Lemma 3. Under the assumptions of Theorem 1, we have

$$
\mathbb{E}\left(\sup _{t \in \Sigma_{m}+\Sigma_{\hat{m}},\|t\|_{L+U}=1} \nu_{n}^{2}(t)-p(m, \hat{m})\right)_{+} \leq \frac{c}{n}
$$

where $p\left(m, m^{\prime}\right)=2\left(m+m^{\prime}\right) / n$.

Therefore $\forall m, m^{\prime}, \quad 4 p\left(m, m^{\prime}\right)-\operatorname{pen}\left(m^{\prime}\right) \leq \operatorname{pen}(m)$ provided that $\operatorname{pen}(m)=\kappa m / n$ with $\kappa \geq 8$. Thus we get, $\forall m \in \mathcal{M}_{n}, \forall S_{m} \in \Sigma_{m}$

$$
\mathbb{E}\left(\left\|\widehat{S}_{\hat{m}}-S_{I}\right\|_{n}^{2} \mathbb{1}_{\Omega_{m}}\right) \leq 3\left\|S_{m}-S_{I}\right\|_{L+U}^{2}+4 \operatorname{pen}(m)+\frac{2 c}{n} .
$$

On the other hand, we need to propose a rough bound for $\left\|\widehat{S}_{\hat{m}}-S_{I}\right\|_{n}^{2}$ in order to control this term on the set $\Omega_{n}^{c}$. To that aim, we prove the Lemma

Lemma 4. Under the Assumption of Theorem 3, for all $m \in \mathcal{M}_{n},\left\|\widehat{S}_{m}-S_{I}\right\|_{n}^{2} \leq 18$, almost surely.

It follows from Lemma 4 and Lemma 2, that

$$
\mathbb{E}\left(\left\|\widehat{S}_{\hat{m}}-S_{I}\right\|_{n}^{2} \mathbb{1}_{\left(\Omega_{n}\right)^{c}}\right) \leq 3 \sqrt{2 \mathbb{P}\left[\left(\Omega_{n}\right)^{c}\right]} \leq \frac{c^{*}}{n},
$$

where $c^{*}$ is a constant. Gathering (28) and (29) gives the first step of the result.

Proof of Lemma 3. We obtain the result by applying the following Theorem: 
Theorem 2. Consider $n \in \mathbb{N}^{*}, \mathcal{F}$ a class at most countable of measurable functions, and $\left(X_{i}\right)_{i \in\{1, \ldots, n\}}$ a family of real independent random variables. Define, for $f \in \mathcal{F}, \nu_{n}(f)=$ $(1 / n) \sum_{i=1}^{n}\left(f\left(X_{i}\right)-\mathbb{E}\left[f\left(X_{i}\right)\right]\right)$, and assume that there are three positive constants $M, H$ and $v$ such that $\sup _{f \in \mathcal{F}}\|f\|_{\infty} \leq M, \mathbb{E}\left[\sup _{f \in \mathcal{F}}\left|\nu_{n}(f)\right|\right] \leq H$, and $\sup _{f \in \mathcal{F}}(1 / n) \sum_{i=1}^{n} \operatorname{Var}\left(f\left(X_{i}\right)\right) \leq v$. Then for all $\alpha>0$,

$$
\mathbb{E}\left[\left(\sup _{f \in \mathcal{F}}\left|\nu_{n}(f)\right|^{2}-2(1+2 \alpha) H^{2}\right)_{+}\right] \leq \frac{4}{b}\left(\frac{v}{n} e^{-b \alpha \frac{n H^{2}}{v}}+\frac{49 M^{2}}{b C^{2}(\alpha) n^{2}} e^{-\frac{\sqrt{2} b C(\alpha) \sqrt{\alpha}}{7} \frac{n H}{M}}\right)
$$

with $C(\alpha)=(\sqrt{1+\alpha}-1) \wedge 1$, and $b=\frac{1}{6}$.

By density arguments, this result can be extended to the case where $\mathcal{F}$ is a unit ball of a linear normed space, after checking that $f \rightarrow \nu_{n}(f)$ is continuous and $\mathcal{F}$ contains a countable dense family.

For our process, we first note that, the collection of models being nested $\Sigma_{m}+\Sigma_{m^{\prime}}=\Sigma_{m \vee m^{\prime}}$. Let $\bar{\varphi}_{j}$ be a linear transformation of the basis $\left(\varphi_{j}\right)_{j}$ orthonormal with respect to the scalar product weighted by $f_{U}+f_{L}$ (by Gramm-Schmidt orthonormalisation), then

$$
\begin{aligned}
\mathbb{E}\left(\sup _{t \in \Sigma_{m}+\Sigma_{m^{\prime}},\|t\|_{L+U}=1} \nu_{n}^{2}(t)\right) & \leq \sum_{j=1}^{m \vee m^{\prime}} \mathbb{E}\left(\nu_{n}^{2}\left(\bar{\varphi}_{j}\right)\right)=\frac{1}{n} \sum_{j=1}^{m \vee m^{\prime}} \operatorname{Var}\left(\bar{\varphi}_{j}\left(L_{1}\right) \varepsilon^{(L)}\left(L_{1}\right)+\bar{\varphi}_{j}\left(U_{1}\right) \varepsilon^{(U)}\left(U_{1}\right)\right) \\
& \leq \frac{2}{n} \sum_{j=1}^{m \vee m^{\prime}} \mathbb{E}\left(\bar{\varphi}_{j}^{2}\left(L_{1}\right)\left(\mathbb{1}_{\delta_{1} \neq-1}-S\left(L_{1}\right)\right)^{2}+\bar{\varphi}_{j}^{2}\left(U_{1}\right)\left(\mathbb{1}_{\delta_{1}=1}-S\left(U_{1}\right)\right)^{2}\right) \\
& \leq \frac{2}{n} \sum_{j=1}^{m \vee m^{\prime}} \mathbb{E}\left(S\left(L_{1}\right)\left(1-S\left(L_{1}\right)\right) \bar{\varphi}_{j}^{2}\left(L_{1}\right)+S\left(U_{1}\right)\left(1-S\left(U_{1}\right)\right) \bar{\varphi}_{j}^{2}\left(U_{1}\right)\right) \\
& \leq \frac{m \vee m^{\prime}}{2 n} \leq \frac{m+m^{\prime}}{2 n}:=H^{2}
\end{aligned}
$$

using that $x(1-x) \leq 1 / 4$ for any $x \in[0,1]$ and $\mathbb{E}\left(\bar{\varphi}_{j}^{2}\left(U_{1}\right)+\bar{\varphi}_{j}^{2}\left(L_{1}\right)\right)=1$ by definition of $\bar{\varphi}_{j}$. Next,

$$
\begin{gathered}
\sup _{t \in \Sigma_{m}+\Sigma_{m^{\prime}},\|t\|_{L+U}=1} \operatorname{Var}\left(t\left(L_{1}\right) \varepsilon^{(L)}\left(L_{1}\right)+t\left(U_{1}\right) \varepsilon^{(U)}\left(U_{1}\right)\right) \leq \\
\sup _{t \in \Sigma_{m}+\Sigma_{m^{\prime}},\|t\|_{L+U}=1} \mathbb{E}\left(t^{2}\left(L_{1}\right)+t^{2}\left(U_{1}\right)\right) \\
=2:=v .
\end{gathered}
$$

Lastly,

$$
\sup _{t \in \Sigma_{m}+\Sigma_{m^{\prime}},\|t\|_{L+U}=1} \sup _{(x, u) \in \mathbb{R}^{+} \times \mathbb{R}^{+}}\left|\varepsilon^{(L)}(x) t(x)+\varepsilon^{(U)}(u) t(u)\right| \leq 2 \sup _{t \in \Sigma_{m}+\Sigma_{m^{\prime}},\|t\|_{L+U}=1} \sup _{x \in \mathbb{R}^{+}}|t(x)| .
$$

For $t=\sum_{j=0}^{m-1} a_{j} \varphi_{j}$, we have $\|t\|_{U+L}^{2}={ }^{t} \vec{a} \Psi_{m} \vec{a}=\left\|\sqrt{\Psi_{m}} \vec{a}\right\|_{m}^{2}$, where $\vec{a}={ }^{t}\left(a_{0}, a_{1}, \ldots, a_{n}\right)$. Thus, for any $m$,

$$
\begin{aligned}
\sup _{t \in \Sigma_{m},\|t\|_{U+L}=1} \sup _{x}|t(x)| & \leq c_{\varphi} \sqrt{m} \sup _{\left\|\sqrt{\Psi_{m}} \vec{a}\right\|_{2, m}=1}\|\vec{a}\|_{m} \\
& \leq c_{\varphi} \sqrt{m} \sup _{\|\vec{u}\|_{m}=1} \mid\left\|\sqrt{\Psi_{m}^{-1}} \vec{u}\right\|_{2, m}=c_{\varphi} \sqrt{m} \sqrt{\left\|\Psi_{m}^{-1}\right\|_{\mathrm{op}}} .
\end{aligned}
$$


Using the definition of $\mathcal{M}_{n}$, we have

$$
\sqrt{m} \sqrt{\left\|\Psi_{m}^{-1}\right\|_{\text {op }}} \leq\left(m\left\|\Psi_{m}^{-1}\right\|_{\mathrm{op}}^{2}\right)^{1 / 4} m^{1 / 4} \leq\left(\mathfrak{c} \frac{n}{\log (n)}\right)^{1 / 4} m^{1 / 4} .
$$

Now, we get a bound similar to the one in Comte and Genon-Catalot (2018) (with $k_{n}=2$ ) and

$$
M_{1}=2 c_{\varphi}\left(\mathfrak{c} \frac{n}{\log (n)}\right)^{1 / 4}\left(m \vee m^{\prime}\right)^{1 / 4} .
$$

Therefore, applying Talagrand inequality recalled in Theorem 2 gives

$$
\begin{aligned}
\mathbb{E}\left(\sup _{t \in \Sigma_{m}+\Sigma_{\hat{m}},\|t\|_{L+U}=1} \nu_{n}^{2}(t)-p(m, \hat{m})\right)_{+} & \leq \sum_{m^{\prime} \in \mathcal{M}_{n}} \mathbb{E}\left(\sup _{t \in \Sigma_{m}+\Sigma_{m^{\prime}},\|t\|_{L+U}=1} \nu_{n}^{2}(t)-p\left(m, m^{\prime}\right)\right)_{+} \\
\leq \sum_{m^{\prime} \in \mathcal{M}_{n}} & \frac{C_{1}}{n}\left(e^{-C_{2}\left(m \vee m^{\prime}\right)}+\frac{\left(m \vee m^{\prime}\right)^{1 / 2}}{n^{1 / 2}} e^{-C_{3}\left(n\left(m \vee m^{\prime}\right)\right)^{1 / 4}}\right) \\
& \leq \frac{C_{4}}{n},
\end{aligned}
$$

for $C_{i}, i=1, \ldots, 4$ constants and $p\left(m, m^{\prime}\right)=4 H^{2}(\alpha=1 / 2)$. This ends the proof.

Proof of Lemma 4. First recall that $\left\|\widehat{S}_{\hat{m}}-S_{I}\right\|_{n}^{2}=\left\|\widehat{S}_{\hat{m}}-S_{I}\right\|_{n, L}^{2}+\left\|\widehat{S}_{\hat{m}}-S_{I}\right\|_{n, U}^{2}$ and we prove that the first term is bounded by 3 , the other term being similar. Now we consider the euclidean norm and recalling the definition of the estimator and of $\Theta_{m}$, we have, for any $m \leq n$ :

$$
\begin{aligned}
n\left\|\widehat{S}_{m}-S_{I}\right\|_{n, L}^{2} & =\left\|\Phi_{m}^{(L)} \Theta_{m}^{-1}\left({ }^{t} \Phi_{m}^{(L)} \vec{\delta}^{(L)}+{ }^{t} \Phi_{m}^{(U)} \vec{\delta}^{(U)}\right)-\vec{S}_{I}(L)\right\|_{2, n}^{2} \\
& \leq 3\left[\left\|\Phi_{m}^{(L)} \Theta_{m}^{-1}{ }^{t} \Phi_{m}^{(L)} \vec{\delta}^{(L)}\right\|_{2, n}^{2}+\left\|\Phi_{m}^{(L)} \Theta_{m}^{-1}{ }^{t} \Phi_{m}^{(U)} \vec{\delta}^{(U)}\right\|_{2, n}^{2}+\left\|\vec{S}_{I}(L)\right\|_{2, n}^{2}\right]
\end{aligned}
$$

with $\vec{S}_{I}(L)={ }^{t}\left(S_{I}\left(L_{1}\right), \ldots, S_{I}\left(L_{n}\right)\right)$. Now we prove that each of the three terms is smaller than or equal to $n$. Clearly, this is true for $\left\|\vec{S}_{I}(L)\right\|_{2, n}^{2}=S_{I}^{2}\left(L_{1}\right)+\cdots+S_{I}^{2}\left(L_{n}\right) \leq n$. Next, by definition of the operator norm, it follows that

$$
\left\|\Phi_{m}^{(L)} \Theta_{m}^{-1}{ }^{t} \Phi_{m}^{(L)} \vec{\delta}^{(L)}\right\|_{2, n}^{2} \leq\left\|\Phi_{m}^{(L)} \Theta_{m}^{-1 t^{t}} \Phi_{m}^{(L)}\right\|_{\mathrm{op}}^{2}\left\|\vec{\delta}^{(L)}\right\|_{2, n}^{2} .
$$

Since $\Phi_{m}^{(L)} \Theta_{m}^{-1}{ }^{t} \Phi_{m}^{(L)}$ is a symmetric positive definite matrix, its operator norm corresponds to its largest eigenvalue. Let $\lambda$ be any eigenvalue of $\Phi_{m}^{(L)} \Theta_{m}^{-1}{ }^{t} \Phi_{m}^{(L)}$ associated with an eigenvector $\vec{x}$ : $\Phi_{m}^{(L)} \Theta_{m}^{-1}{ }^{t} \Phi_{m}^{(L)} \vec{x}=\lambda \vec{x}$. Multiplying both sides by ${ }^{t} \Phi_{m}^{(L)}$, we get, for $\vec{y}={ }^{t} \Phi_{m}^{(L)} \vec{x},{ }^{t} \Phi_{m}^{(L)} \Phi_{m}^{(L)} \Theta_{m}^{-1} \vec{y}=$ $\lambda \vec{y}$, which means that $\lambda$ is an eigenvalue of ${ }^{t} \Phi_{m}^{(L)} \Phi_{m}^{(L)} \Theta_{m}^{-1}$. Now setting $\vec{z}=\left({ }^{t} \Phi_{m}^{(L)} \Phi_{m}^{(L)}\right)^{-1 / 2} \vec{y}$ where $S^{1 / 2}$ is a symmetric square root of a symmetric matrix $S$, we obtain that $\lambda$ is also an eigenvalue of

$$
\left({ }^{t} \Phi_{m}^{(L)} \Phi_{m}^{(L)}\right)^{1 / 2} \Theta_{m}^{-1}\left({ }^{t} \Phi_{m}^{(L)} \Phi_{m}^{(L)}\right)^{1 / 2}=\left[\operatorname{Id}_{m}+\left({ }^{t} \Phi_{m}^{(L)} \Phi_{m}^{(L)}\right)^{-1 / 2}\left({ }^{t} \Phi_{m}^{(U)} \Phi_{m}^{(U)}\right)\left({ }^{t} \Phi_{m}^{(L)} \Phi_{m}^{(L)}\right)^{-1 / 2}\right]^{-1},
$$

where $\operatorname{Id}_{m}$ is the $m \times m$ identity matrix. Clearly $M:=\left({ }^{t} \Phi_{m}^{(L)} \Phi_{m}^{(L)}\right)^{-1 / 2}\left({ }^{t} \Phi_{m}^{(U)} \Phi_{m}^{(U)}\right)\left({ }^{t} \Phi_{m}^{(L)} \Phi_{m}^{(L)}\right)^{-1 / 2}$ is symmetric positive definite and is diagonalizable in an orthonormal basis as $\operatorname{diag}\left(a_{1}, \ldots, a_{m}\right)$ with $a_{i}>0$ for $i=1, \ldots, m$. In this basis $(I+M)^{-1}$ is equal to $\left(1 /\left(1+a_{1}\right), \ldots, 1 /\left(1+a_{m}\right)\right)$, and all these eigenvalues are in $(0,1)$. Therefore $\lambda \leq 1$ and thus $\left\|\Phi_{m}^{(L)} \Theta_{m}^{-1}{ }^{t} \Phi_{m}^{(L)}\right\|_{\text {op }}^{2} \leq 1$. Consequently, using that all the coordinates of $\vec{\delta}^{(L)}$ belong to $[-1,1]$, we get the second bound

$$
\left\|\Phi_{m}^{(L)} \Theta_{m}^{-1}{ }^{t} \Phi_{m}^{(L)} \vec{\delta}^{(L)}\right\|_{2, n}^{2} \leq\left\|\vec{\delta}^{(L)}\right\|_{2, n}^{2} \leq n .
$$


For the last term, we also start with

$$
\left\|\Phi_{m}^{(L)} \Theta_{m}^{-1}{ }^{t} \Phi_{m}^{(U)} \vec{\delta}^{(U)}\right\|_{2, n}^{2} \leq\left\|\Phi_{m}^{(L)} \Theta_{m}^{-1}{ }^{t} \Phi_{m}^{(U)}\right\|_{\text {op }}^{2}\left\|\vec{\delta}^{(U)}\right\|_{2, n}^{2} .
$$

Here the matrix $\Phi_{m}^{(L)} \Theta_{m}^{-1}{ }^{t} \Phi_{m}^{(U)}$ is not symmetric and thus

$$
\left\|\Phi_{m}^{(L)} \Theta_{m}^{-1}{ }^{t} \Phi_{m}^{(U)}\right\|_{\text {op }}^{2}=\lambda_{\max }\left(\Phi_{m}^{(L)} \Theta_{m}^{-1}{ }^{t} \Phi_{m}^{(U)} \Phi_{m}^{(U)} \Theta_{m}^{-1} t^{(L)} \Phi_{m}^{(L)}\right)
$$

where $\lambda_{\max }(A)$ stands for the largest eigenvalue of a matrix $A$ and $\|A\|_{\text {op }}^{2}=\lambda_{\max }\left({ }^{t} A A\right)$. As previously an eigenvalue of $\Phi_{m}^{(L)} \Theta_{m}^{-1}{ }^{t} \Phi_{m}^{(U)} \Phi_{m}^{(U)} \Theta_{m}^{-1}{ }^{t} \Phi_{m}^{(L)}$ is also an eigenvalue of

$$
\left(\operatorname{Id}_{m}+{ }^{t} \Phi_{m}^{(U)} \Phi_{m}^{(U)}\left({ }^{t} \Phi_{m}^{(L)} \Phi_{m}^{(L)}\right)^{-1}\right)^{-1}\left(\operatorname{Id}_{m}+{ }^{t} \Phi_{m}^{(L)} \Phi_{m}^{(L)}\left({ }^{t} \Phi_{m}^{(U)} \Phi_{m}^{(U)}\right)^{-1}\right)^{-1}
$$

as both matrices are equal (note that ${ }^{t} \Phi_{m}^{(U)} \Phi_{m}^{(U)}\left({ }^{t} \Phi_{m}^{(L)} \Phi_{m}^{(L)}\right)^{-1}$ is the inverse of $\left.{ }^{t} \Phi_{m}^{(L)} \Phi_{m}^{(L)}\left({ }^{t} \Phi_{m}^{(U)} \Phi_{m}^{(U)}\right)^{-1}\right)$. Consider a basis in which the first one is diagonal and of the form $\operatorname{Diag}\left(a_{1}, \ldots, a_{m}\right)$, then the whole matrix is of the form $\operatorname{Diag}\left(1 /\left[\left(1+a_{1}\right)\left(1+a_{1}^{-1}\right)\right], \ldots, 1 /\left[\left(1+a_{m}\right)\left(1+a_{m}^{-1}\right)\right]\right)$, that is the eigenvalues are less that 1 as soon as the $a_{i}$ 's are positive. Now let $a$ be an eigenvalue with $\vec{x}$ associated eigenvector, that is ${ }^{t} \Phi_{m}^{(U)} \Phi_{m}^{(U)}\left({ }^{t} \Phi_{m}^{(L)} \Phi_{m}^{(L)}\right)^{-1} \vec{x}=a \vec{x}$. Then ${ }^{t} \Phi_{m}^{(U)} \Phi_{m}^{(U)} \vec{y}=a^{t} \Phi_{m}^{(L)} \Phi_{m}^{(L)} \vec{y}$ and taking the scalar product with $\vec{y}$ yields

$$
{ }^{t} \vec{y}^{t} \Phi_{m}^{(U)} \Phi_{m}^{(U)} \vec{y}=a^{t^{t}}{ }^{t} \Phi_{m}^{(L)} \Phi_{m}^{(L)} \vec{y}
$$

that is $\left\|\Phi_{m}^{(U)} \vec{y}\right\|_{2, n}^{2}=a\left\|\Phi_{m}^{(L)} \vec{y}\right\|_{2, n}^{2}$. Thus $a \geq 0$. Lastly $a \neq 0$ because of invertibility assumptions. We obtain that $\left\|\Phi_{m}^{(L)} \Theta_{m}^{-1}{ }^{t} \Phi_{m}^{(U)}\right\|_{\text {op }}^{2} \leq 1$ and thus

$$
\left\|\Phi_{m}^{(L)} \Theta_{m}^{-1 t^{t}} \Phi_{m}^{(U)} \vec{\delta}^{(U)}\right\|_{2, n}^{2} \leq n .
$$

Therefore, gathering the three bounds $n$ for the euclidean norms gives the bound 9 for the empirical norm and ends the proof.

6.5. Proof of Inequality (18). We already mentioned that ${ }^{t} \vec{v} \Psi_{m, Z} \vec{v}=\int_{I} v^{2}(x) f_{Z}(x) d x$ for $Z=L, U$ and $v(x)=\sum_{j=0}^{m-1} v_{j} \varphi_{j}(x)$ where $\vec{v}={ }^{t}\left(v_{0}, \ldots, v_{m-1}\right)$. Thus if $\forall x \in I, f_{Z}(x) \geq f_{0}$, we get for any vector $\vec{v} \in \mathbb{R}^{d}$,

$$
{ }^{t} \vec{v} \Psi_{m, Z} \vec{v} \geq f_{0} \int_{I} v^{2}(x) d x=f_{0} \sum_{j=0}^{m-1} v_{j}^{2} .
$$

As a consequence, for any vector $\vec{v} \in \mathbb{R}^{d}$,

$$
{ }^{t_{\vec{v}}}\left(\Psi_{m, L}+\Psi_{m, U}\right) \vec{v} \geq 2 f_{0} \int_{I} v^{2}(x) d x=2 f_{0} \sum_{j=0}^{m-1} v_{j}^{2} .
$$

all eigenvalues of $\Psi_{m, L}+\Psi_{m, U}$ are larger than $2 f_{0}$ and therefore, as they are all positive, the largest eigenvalue of $\left(\Psi_{m, L}+\Psi_{m, U}\right)^{-1}$ is smaller that $1 /\left(2 f_{0}\right)$.

\section{REFERENCES}

Abramowitz, M. and Stegun, I. A. (1964), Handbook of mathematical functions: with formulas, graphs, and mathematical tables, Vol. 55, Courier Corporation.

Anderson-Bergman, C. and Yu, Y. (2016), 'Computing the log concave npmle for interval censored data', Statistics and Computing 26(4), 813-826.

Baraud, Y. (2002), 'Model selection for regression on a random design', ESAIM: Probability and Statistics 6, 127-146. 
Baudry, J.-P., Maugis, C. and Michel, B. (2012), 'Slope heuristics: overview and implementation', Statistics and Computing 22(2), 455-470.

Becker, N. G. and Melbye, M. (1991), 'Use of a log-linear model to compute the empirical survival curve from interval-censored data, with application to data on tests for hiv positivity', Australian Journal of Statistics 33(2), 125-133.

Birgé, L. (1999), 'Interval censoring: a nonasymptotic point of view', Mathematical Methods of Statistics 8(3), 285-298.

Bongioanni, B. and Torrea, J. L. (2009), 'What is a sobolev space for the laguerre function systems?', Studia Mathematica 192, 147-172.

Braun, J., Duchesne, T. and Stafford, J. E. (2005), 'Local likelihood density estimation for interval censored data', Canadian Journal of Statistics 33(1), 39-60.

Brunel, E. and Comte, F. (2009), 'Cumulative distribution function estimation under interval censoring case 1', Electronic journal of statistics 3, 1-24.

Carstensen, B. (1996), 'Regression models for interval censored survival data: application to hiv infection in danish homosexual men', Statistics in Medicine 15(20), 2177-2189.

Chernozhukov, V., Fernandez-Val, I. and Galichon, A. (2009), 'Improving point and interval estimators of monotone functions by rearrangement', Biometrika 96(3), 559-575.

Comte, F. and Dion, C. (2017), 'Laguerre estimation under constraint at a single point', Preprint MAP5 2017-04 and hal-01447605.

Comte, F. and Genon-Catalot, V. (2018), 'Regression function estimation on non compact support as a partly inverse problem', Preprint MAP5 2018-01 and hal-01690856v2.

Comte, F., Genon-Catalot, V. et al. (2015), 'Adaptive laguerre density estimation for mixed poisson models', Electronic Journal of Statistics 9(1), 1113-1149.

Geskus, R. B. and Groeneboom, P. (1996), 'Asymptotically optimal estimation of smooth functionals for interval censoring, part 1', Statistica Neerlandica 50(1), 69-88.

Geskus, R. B. and Groeneboom, P. (1997), 'Asymptotically optimal estimation of smooth functionals for interval censoring, part 2', Statistica Neerlandica 51(2), 201-219.

Geskus, R., Groeneboom, P. et al. (1999), 'Asymptotically optimal estimation of smooth functionals for interval censoring, case 2', The Annals of Statistics 27(2), 627-674.

Groeneboom, P. and Ketelaars, T. (2011), 'Estimators for the interval censoring problem', Electronic Journal of Statistics 5, 1797-1845.

Groeneboom, P. and Wellner, J. A. (1992), Information bounds and nonparametric maximum likelihood estimation, Vol. 19, Springer Science; Business Media.

Kooperberg, C. and Stone, C. J. (1992), 'Logspline density estimation for censored data', Journal of Computational and Graphical Statistics 1(4), 301-328.

Melbye, M., Biggar, R. J., Ebbesen, P., Sarngadharan, M., Weiss, S. H., Gallo, R. C. and Blattner, W. A. (1984), 'Seroepidemiology of htlv-iii antibody in danish homosexual men: prevalence, transmission, and disease outcome.', British Medical Journal (Clinical Research Edition) 289(6445), 573-575.

Tropp, J.A. (2015), 'An introduction to matrix concentration inequalities', Found. Trends Mach. Learn., 8(1-2):1-230.

Turnbull, B. W. (1976), 'The empirical distribution function with arbitrarily grouped, censored and truncated data', Journal of the Royal Statistical Society. Series B (Methodological) pp. 290-295.

Yang, S. (2000), 'Functional estimation under interval censoring case 1', Journal of statistical planning and inference 89(1), 135-144. 


\section{Appendix A. Numerical Results}

We give in Table 1 the numerical results corresponding to the simulations and boxplots of Section 4. We add the mean squared errors computed for the empirical survival function evaluated with the whole sample $X_{i}$, as a benchmark or a kind of "oracle" estimator which represents the best we could obtain if we had observed directly the $X_{i}^{\prime} s$ without censoring, instead of the intervals $\left[L_{i}, U_{i}\right]$.

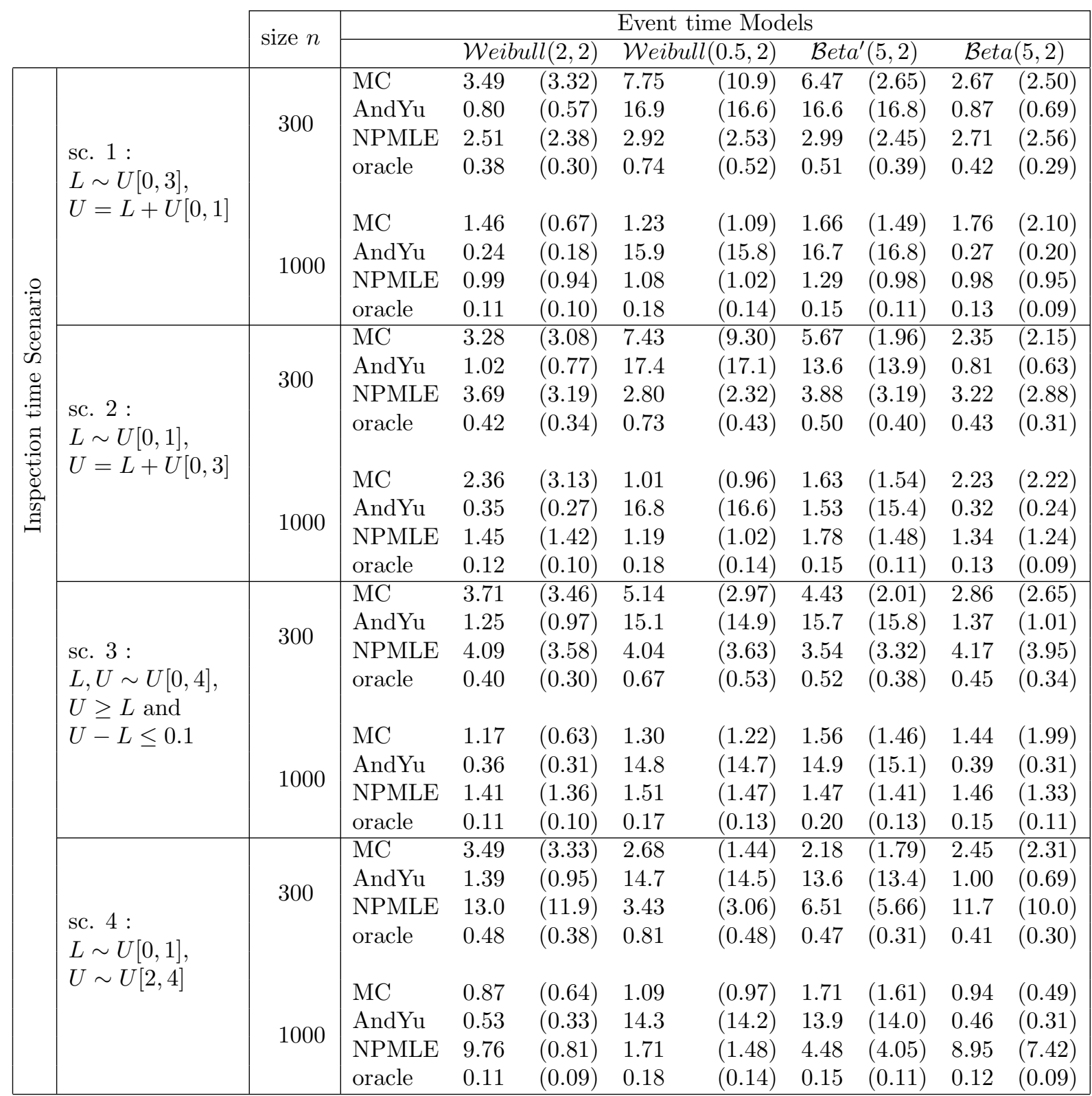

TABLE 1. Average Mean Squared Error AMSE $\times 10^{-3}$ and Median in parenthesis for our penalized Least Squares estimator built with Laguerre basis (MC), the log-concave Anderson-Bergman and Yu's NPMLE (AndYu), the unconstrained NPMLE (NPMLE) and the "oracle" empirical survival function. 